\title{
Diffusion, diffraction des neutrons en temps réel et études réalisées in situ
}

\author{
O. Isnard
}

Université J. Fourier, Grenoble I, Laboratoire de Cristallographie, UPR 5031 du CNRS, BP. 166X, 38042 Grenoble cedex 9, France

\section{INTRODUCTION}

II. DEUX MOTS DE TERMINOLOGIE

III. DIFFERENTS TYPES DE MESURES : METHODOLOGIE

III.a. Processus irréversibles : mesures simples

III.b. Processus réversibles : mesures stroboscopiques

IV. LA DIFFRACTION DE POUDRE IN SITU RESOLUE EN TEMPS

IV.a. PRINCIPES

IV.b. LA THERMODIFFRACTION

IV.b.1. Etude de la cristallisation de phase amorphe

IV.b.2. Etude de transition de phase structurale

IV.b.3 Etude de transition de phase magnétique

IV.c. Etudes en temps réel et in situ de réactions chimiques

IV.c.1. Etude de réaction solide-gaz

IV.c.2. Analyse électrochimique in situ

IV.c.3. Réaction d'intercalations chimiques dans le graphite

IV.c.4. Déshydratation

IV.c.5. Synthèse de composé

IV.d. Informations structurales contenues en dehors des pics de Bragg

IV.e. Analyse des données de diffraction résolue dans le temps

V. ANALYSES EN TEMPS REEL PAR DIFFUSION AUX PETITS ANGLES

VI. SPECTROMETRIE TROIS AXES RESOLUE EN TEMPS

VII. LA DIFFRACTION RESOLUE EN TEMPS ET IN SITU SUR MONOCRISTAL

VIII. LIMTTATIONS DE LA DIFFRACTION RESOLUE EN TEMPS

VIII.a. Echelle de temps caractéristique

VIII.b. Développements en cours

IX. CONCLUSIONS

X. REFERENCES

\section{Résumé}

La diffusion des neutrons est une technique particulièrement efficace pour l'analyse en temps réel des processus réactionnels dans la matière. La diffraction de neutrons in situ a été développée très tôt sur les sources à haut flux tel que l'Institut Laue Langevin. Ces études nécessitent un flux de neutrons important et un détecteur couvrant un domaine angulaire le plus grand possible. Les neutrons offrent la spécificité d'être très peu absorbés par nombre de matériaux, cette faible absorption fait de la diffusion neutronique un excellent outil pour sonder la matière en volume et de manière non destructive. Cela permet en particulier d'utiliser des 
environnements d'échantillons complexes tout en conservant un flux raisonnable. La diffusion de neutrons en temps réel est donc très largement utilisée par diverses communautés scientifiques : sciences des matériaux, physiciens, chimistes...

L'objet de ce cours est de donner les paramètres importants pour ce type d'étude et d'illustrer le propos à l'aide d'exemples pris dans des domaines scientifiques divers: électrochimie, magnétisme, métallurgie, chimie du solide. Après avoir présenté quelques repères méthodologiques sur les méthodes d'acquisition de données, des exemples montreront le fort potentiel de la diffusion neutronique en temps réel pour l'étude de la matière dans des conditions dynamiques. Enfin, nous donnerons aussi quelques conseils pour la visualisation, le dépouillement et l'analyse de ce type d'expérience.

La diffusion des neutrons sur poudre est actuellement très bien adaptée aux études réalisées in situ. Cependant, nous verrons que la faisabilité d'études in situ s'étend à d'autres techniques expérimentales telles que la diffusion des neutrons aux petits angles et même la diffusion sur monocristal qui est en plein renouveau.

\section{INTRODUCTION}

Longtemps, les techniques de diffraction des rayons $\mathrm{X}$ ou de neutrons ont été limitées à l'étude structurale et microstructurale d'échantillon cristallin ou non mais en tout cas à des études statiques. En effet, la durée d'acquisition des données expérimentales, qui était de l'ordre de l'heure ou de la journée, ne permettait pas l'étude de cinétique de réaction.

Le développement de nouveaux détecteurs mais aussi l'apparition de nouvelles sources, plus intenses (anticathode tournante, rayonnement synchrotron, réacteur de neutrons à haut flux, source à spallation) permettent désormais d'appréhender le suivi en temps réel des mécanismes de transition de phase.

Au fil de cet article, nous décrirons la méthodologie à mettre en œuvre pour effectuer des expériences de diffusion de neutrons en temps réel. Ensuite, c'est la diffraction neutronique sur poudre résolue en temps qui sera décrite, d'abord dans son principe puis au travers d'exemples divers de physique comme de chimie. Quelques mots sont aussi dédiés à la description de la méthode d'analyse des données de diffraction résolue en temps. Puis, d'autres techniques de diffusion de neutrons en temps réel seront abordées avec des exemples : diffusion de neutrons aux petits angles, spectrométrie trois axes, diffusion sur monocristal. Enfin, après une présentation des échelles de temps caractéristiques, les limites actuelles de la diffraction résolue en temps seront discutées ainsi que les développements futurs.

\section{DEUX MOTS DE TERMINOLOGIE}

Il convient tout d'abord de distinguer les études réalisées in situ de celles menées en temps réel. Les travaux menés in situ peuvent être effectués dans un environnement d'échantillon plus ou moins complexe voire encombrant (pression élevée, température extrême, cellule réactionnelle, ex : cellule électrochimique etc...) mais ne nécessite pas forcément le suivi en temps réel d'un processus réactionnel physique ou chimique dépendant du temps. On peut ainsi effectuer quelques mesures ( 2,3 ou plus) dans des conditions expérimentales définies (pression, température, composition) afin de déterminer un diagramme de phase. Il est cependant possible d'effectuer à la fois des expériences in situ et en temps réel. Cela permet bien souvent de progresser de manière significative dans la compréhension des mécanismes mis en jeu lors des transformations de la matière condensée. Dans la suite de cet exposé, les termes "résolue en temps » et "en temps réel » seront utilisés indifféremment. 


\section{DIFFERENTS TYPES DE MESURES : METHODOLOGIE}

Selon le type d'analyse à effectuer, deux stratégies de collecte peuvent être mises en œuvre. Dans le cas des processus irréversibles, il est indispensable de réaliser une acquisition de manière séquentielle et en temps réel. $\mathrm{Si}$, en revanche, le temps de réaction est trop court pour qu'un diagramme exploitable soit enregistré mais que le processus est reproductible, il est alors envisageable de le reproduire de façon cyclique et d'effectuer des mesures stroboscopiques : c'est la stratégie la mieux adaptée pour l'étude d'un processus réversible et particulièrement rapide.

\section{III.a. Processus irréversibles : mesures simples}

Dans ce cas, le système physique (échantillon) est mis dans un état métastable et l'on va suivre, grâce à une acquisition séquentielle des diagrammes, l'évolution de l'échantillon. Le déclenchement du processus peut être réalisé de diverses façons. Tout d'abord en changeant une variable thermodynamique (température, pression, champ magnétique...). Cette perturbation peut être effectuée de manière continue (cf exemple $c$ de la figure 1); c'est le cas de la thermodiffractométrie (variation continue de la température) qui sera détaillée plus loin. Mais il est aussi possible d'appliquer une impulsion extérieure pour initier la réaction, cas d'une excitation de type a ou b de la figure 1. Cette impulsion peut être lumineuse, électrique ou prendre toute autre forme telle que l'introduction de gaz dans une enceinte etc... Il est aussi envisageable de mélanger différents produits réactifs pour initier une réaction chimique.

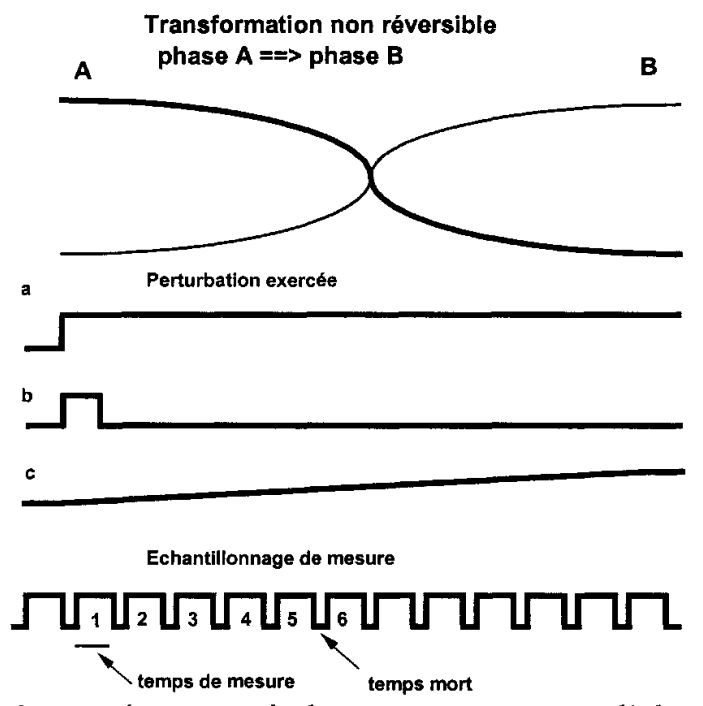

Figure 1 : Principe des expériences résolues en temps pour l'observation de processus irréversibles.

L'excitation ou perturbation sert à déclencher le processus et peut prendre plusieurs formes : discontinue, impulsionnelle ou progressive. Ces formes correspondent respectivement aux cas $\mathrm{a}, \mathrm{b}$ et $\mathrm{c}$ indiqués sur la figure 1 . A partir du moment où la source de neutrons est ouverte, l'acquisition peut être décomposée en deux temps successifs: $t_{m}$ qui représente le temps d'acquisition proprement dit et $t_{c}$ qui est un temps mort correspondant au transfert et au stockage des données. La somme des ces deux temps définit la période d'échantillonnage du processus 
investigué. Idéalement, le temps de mesure doit être réduit par rapport au temps caractéristique du processus suivi. Cependant cela dépend du pouvoir diffusant de l'échantillon, de l'environnement échantillon qui peut lui-même être plus ou moins absorbant, et bien sûr du flux disponible sur l'instrument qui est souvent déterminant.

De nombreuses expériences de ce type seront illustrées dans la suite de ce cours et nous reviendrons dans la partie VIII sur les échelles de temps accessibles selon le type d'expériences effectuées.

\section{III.b. Processus réversibles : mesures stroboscopiques}

Lorsqu'un processus de transformation est réversible mais de durée trop courte pour pouvoir être déterminé par des diagrammes séquentiels en une fois, il est alors possible de répéter la mesure de façon cyclique un grand nombre de fois et d'accumuler les mesures faites dans les mêmes conditions. Ces mesures dites stroboscopiques sont un moyen efficace pour accroître la résolution temporelle. Les mesures stroboscopiques permettent de compenser :

- soit le faible flux,

- soit la rapidité du processus,

- soit la saturation du détecteur qui surviendrait si le flux était plus fort.

Cela nécessite cependant une bonne dynamique des compteurs et de la chaîne de comptage. Il faut une synchronisation parfaite entre le système d'acquisition de données et le phénomène ou l'état de l'échantillon. Comme l'indique la figure 2, l'enregistrement du diagramme est généralement effectué en permanence alors que la perturbation appliquée au système est appliquée de manière répétitive, cyclique. La fréquence d'acquisition peut être significativement augmentée en réduisant le temps $t_{\mathrm{m}}$ à condition d'accumuler suffisamment de diagrammes enregistrés à chaque cycle dans les mêmes conditions. C'est une façon pratique d'améliorer la statistique de comptage en dépit d'un faible temps d'acquisition.
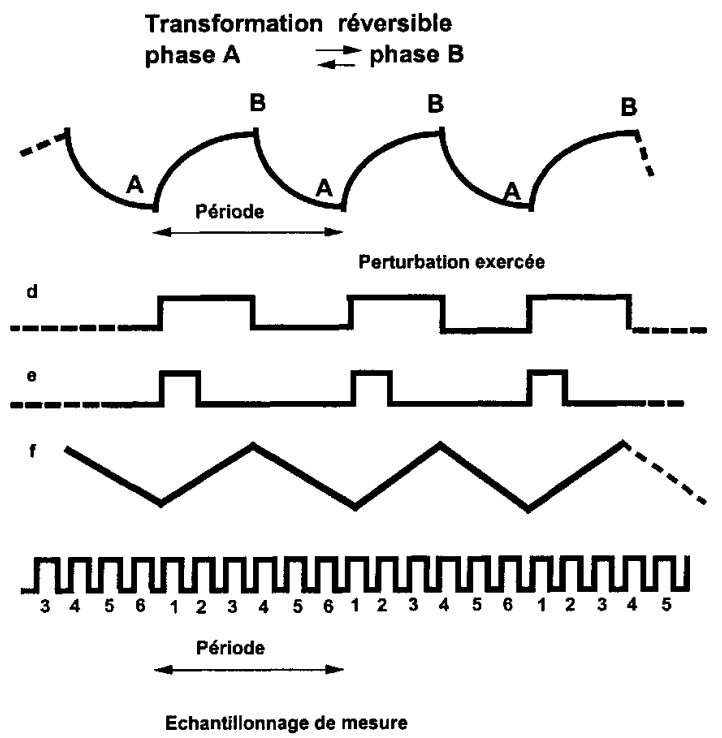

Figure 2 : Principe des expériences résolues en temps pour l'observation de processus réversibles. 
Des mesures stroboscopiques de diffusion de neutrons ont permis d'étudier la cinétique de transition de phase induite par contrainte dans le quartz [Gibhardt 2000]. Ainsi l'application périodique (toutes les 20 secondes) de contraintes uniaxiales (plusieurs dizaines de MPa) sur un échantillon de quartz a permis de suivre en temps réel la transition de phase de la phase haute température $(\beta)$ vers une phase incommensurable. L'évolution de la position des pics de Bragg et des satellites a pu être suivie avec un temps d'échantillonnage inférieur à la seconde et ceci même sur un instrument de flux relativement modeste. La diffusion de neutrons a permis de démontrer que la réponse du cristal de quartz à la sollicitation mécanique est caractérisée par un temps de relaxation de $0,7 \mathrm{~s}$.

\section{LA DIFFRACTION DE POUDRE IN SITU RESOLUE EN TEMPS}

Comparé à leur équivalent statique, le principal avantage des expériences résolues dans le temps réside dans leur potentialité à étudier non plus des échantillons figés mais les processus de transformation de la matière condensée sous l'action de diverses perturbations externes telles que température, réactifs chimiques, etc. Ce type d'expériences de diffraction permet par exemple :

- de mettre en évidence des structures transitoires à courtes durées de vie,

- d'élucider le mécanisme des transformations à partir de la mesure des modifications structurales et microstructurales subies par les cristallites de l'échantillon,

- de mesurer la cinétique des transformations à partir de l'évolution au cours du temps de la fraction massique de chacune des phases cristallisées contenues dans la poudre,

- d'étudier les phénomènes hors équilibres.

\section{IV.a. PRINCIPES}

D'un point de vue expérimental, les études résolues en temps nécessitent de mesurer rapidement et simultanément la plus grande fraction possible de l'espace réciproque. La rapidité de mesure est bien sûr importante, en pratique pour les études de diffraction effectuées en dispersion angulaire; les mesures en temps réel requièrent l'emploi d'un détecteur de localisation fixe et couvrant un large domaine angulaire (voir cours sur les dispositifs expérimentaux dans cette école).

Une autre contrainte expérimentale résulte de l'aspect in situ de ce type d'expérience qui implique la nécessité de contrôler l'environnement physique de l'échantillon et de mesurer très précisément son état (température, pression etc..) durant toute la mesure. L'environnement échantillon luimême peut être encombrant (four, cryostat, cellule réactionnelle etc..). Cet environnement doit cependant rester compatible avec l'optique de diffraction, c'est-à-dire comporter des «fenêtres » nécessaires à l'entrée du faisceau incident et à la sortie du faisceau diffracté. Il faut aussi limiter autant que possible la présence de pics parasites de diffraction générés par l'environnement de l'échantillon. Les propriétés spécifiques du neutron, en particulier sa grande pénétrabilité en font une sonde de choix pour les expériences in situ.

La diffraction dans des conditions dynamiques est apparue grâce à l'usage des sources neutroniques, qui permettent de disposer d'un environnement adéquat pour installer des cellules de réactions diverses. C'est ainsi qu'est née la thermodiffractométrie. Les instruments dédiés à ces études combinent en général deux caractéristiques essentielles: un flux important et un multidétecteur permettant rapidement l'enregistrement de la majeure partie ou de l'intégralité du diagramme de diffraction. L'Institut Laue Langevin, disposant à la fois du plus haut flux de neutrons et de la maîtrise des multidétecteurs développés à Grenoble, fut naturellement à la pointe de cette activité de recherche. Un instrument tel que D1B tire ainsi le meilleur profit de son multidétecteur [Convert 1975, Convert 1983] couvrant un domaine angulaire de $80^{\circ} 20$. A titre 


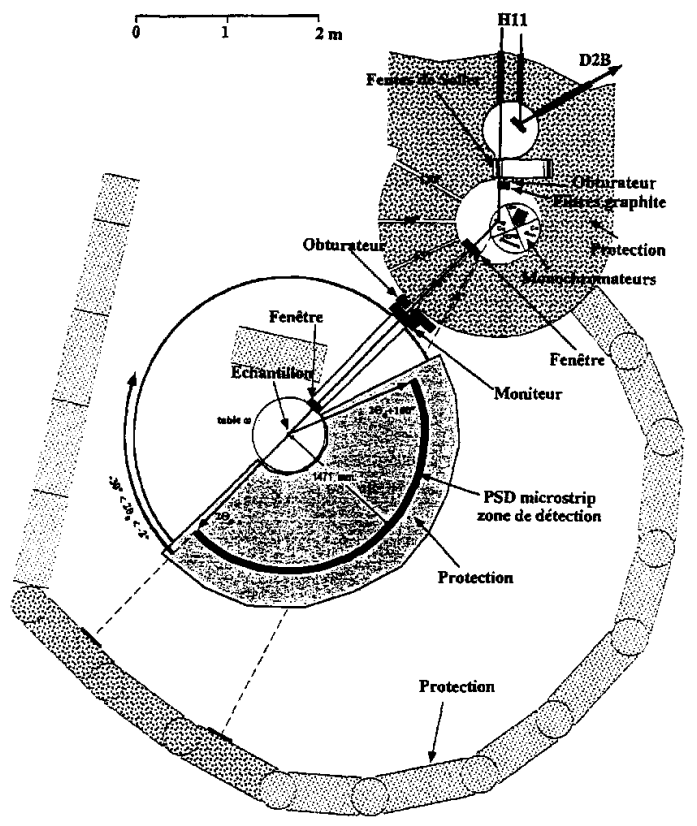

Figure 3 : Représentation schématique de l'instrument D20 de l'Institut Laue Langevin

d'exemple, la figure 3 présente de manière schématique la configuration d'un autre instrument particulièrement bien adapté pour les études en temps réel : le diffractomètre D20 lui aussi à l'Institut Laue Langevin.

Il est temps de citer les articles de synthèse [Pannetier 1986, Pannetier 1988] qui retracent les travaux pionniers en terme de diffusion de neutrons en temps réel et ont eu le mérite de révéler aux physiciens et chimistes du solide la puissance de la technique. Du point de vue des sources de rayons $\mathrm{X}$ de laboratoire, l'apparition de détecteurs à localisation spatiale développés à Grenoble [Ballon et al. 1983] et commercialisés par la société INEL a permis le développement de la thermodiffractométrie dans les laboratoires de recherche. Ce type de détecteur permet l'acquisition simultanée d'un diagramme de poudre sur une zone angulaire de $120^{\circ}(2 \theta)$. Divers laboratoires ont ainsi développé des fours permettant les études en temps réel par thermodiffractométrie $X$ [Barret et al. 1968, Plévert et al 89]. Depuis, la technique a été étendue aux sources synchrotron, mettant alors en avant l'acquisition de données de diffraction dans un laps de temps extrêmement court. Le suivi dynamique d'une réaction explosive a même pu être réalisé [Wong et al 1990].

\section{IV.b. LA THERMODIFFRACTION}

L'une des premières et des plus simples mises en œuvre de la diffraction en temps réel est la thermodiffraction. Elle consiste en un suivi de l'évolution thermique du diagramme de diffraction au cours d'une variation de température. Ainsi, l'établissement d'un diagramme de phase complet peut être facilité par ce type d'analyse, plus exhaustif qu'une analyse de seulement quelques points en température. 


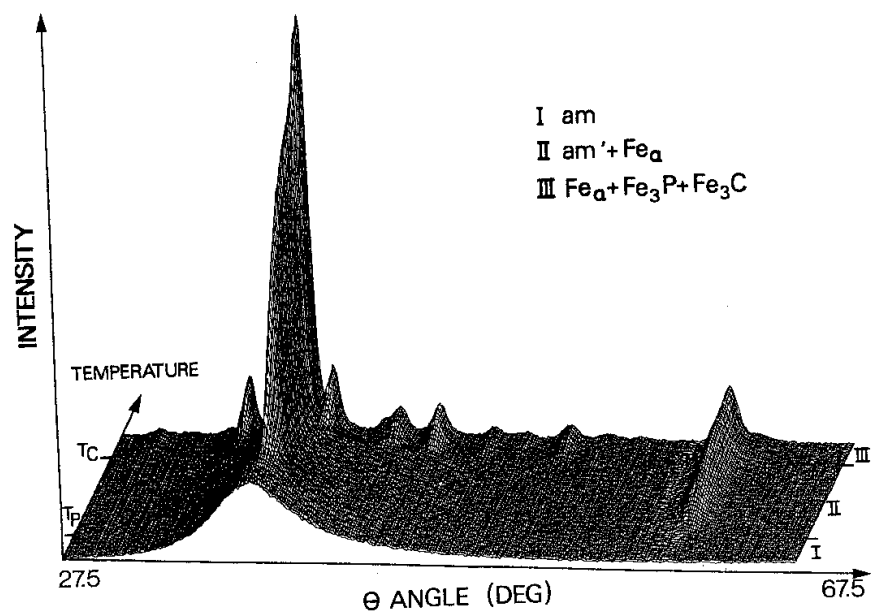

Figure 4 : Evolution thermique du diagramme de diffraction neutronique mesuré in situ d'un acier amorphe en cours de recristallisation.

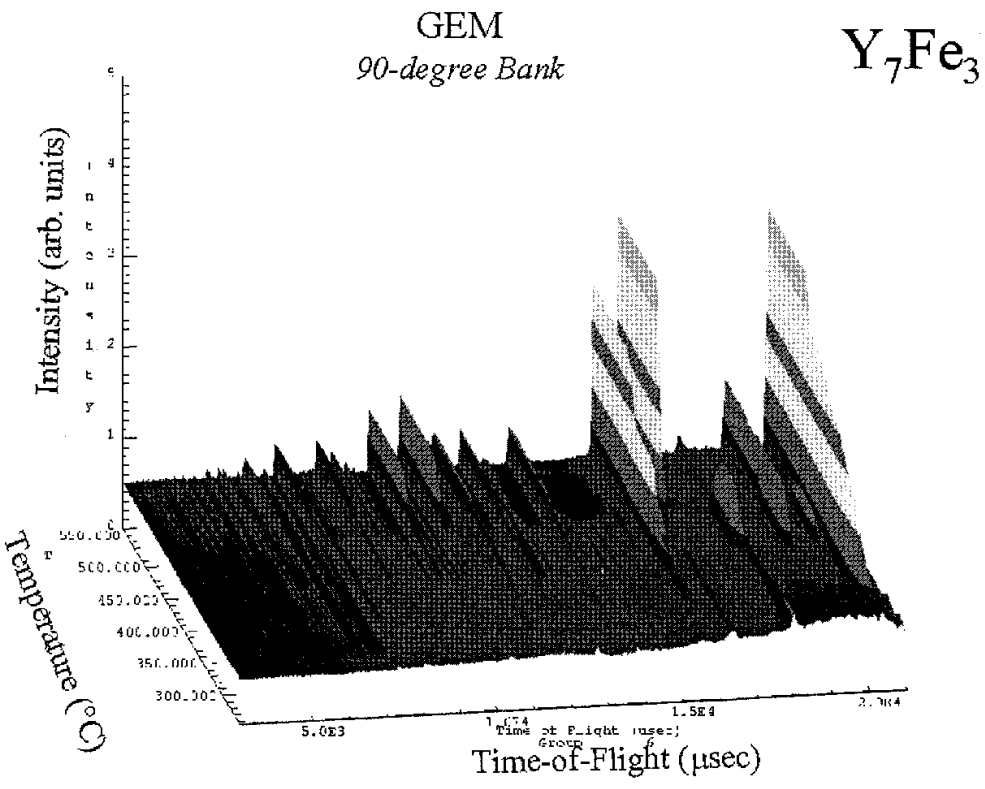

Figure 5: Evolution du diagramme de diffraction neutronique du composé amorphe $Y_{7} \mathrm{Fe}_{3}$ au cours de sa cristallisation [Kilcoyne et al. 2001], mesures effectuées sur une source à spallation, instrument GEM d'ISIS [Williams et al. 1998].

\section{IV.b.1. Etude de la cristallisation de phase amorphe}

Une utilisation désormais courante de la diffraction neutronique consiste à suivre l'évolution d'un matériau amorphe lors de la cristallisation et ainsi de pouvoir déterminer les conditions optimales de synthèse du matériau souhaité. Ce type d'étude a souvent été réalisé à l'I.L.L. sur l'instrument D1B mais est aussi effectué sur D20. 
Un exemple de diagramme mesuré sur un composé métallique amorphe est donné figure 4 . On observe progressivement le passage par trois stades différents : I la présence de la phase amorphe (notée $\mathrm{am}$ ) à basse température, II la coexistence d'une phase amorphe (am') et de fer sous forme $\alpha$, caractérisée par les deux pics de Bragg les plus intenses du diagramme. Enfin, à plus haute température, seules trois phases cristallisées sont présentes dans le diagramme de diffraction neutronique $\mathrm{Fe} \alpha, \mathrm{Fe}_{3} \mathrm{P}$ et $\mathrm{Fe}_{3} \mathrm{C}$. Comme nous venons de le voir, le suivi in situ de la cristallisation d'un verre métallique massif permet de mettre en évidence les processus de mise en ordre du composé. Partant d'un composé amorphe, le diagramme de diffraction neutronique n'est d'abord caractérisé que par des bosses de diffusion, puis on note au fur et à mesure de la réaction que des pics de diffraction apparaissent progressivement. La largeur des pics de Bragg peut aussi s'affiner au cours de la cristallisation progressive du composé. Les neutrons sont ici précieux car ils renseignent directement sur l'ensemble de l'échantillon et permettent de connaître l'état de cristallisation au cœur de la matière.

Une étude comparative de la cristallisation d'un alliage amorphe de composition $\mathrm{Y}_{7} \mathrm{Fe}_{3}$ a été réalisée à la fois sur l'instrument D20 de l'ILL et sur l'instrument GEM de la source à spallation d'ISIS [Williams et al. 98]. Ce type d'étude est typique de l'utilisation de la thermodiffractométrie. Il est donc désormais possible d'effectuer ce type d'analyse sur une source à spallation. Les figures 5 et 6 montrent toutes deux que si le produit final de la cristallisation est la phase de Laves $\mathrm{YFe}_{2}$, une nouvelle phase intermétallique apparaît à des températures intermédiaires.

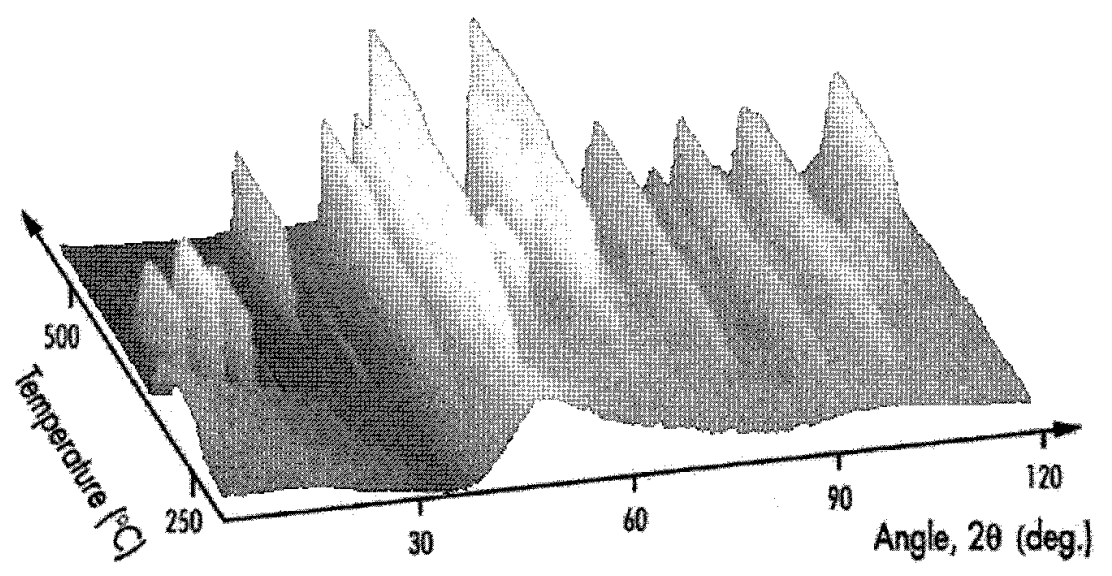

D20 ILL

Figure 6: Evolution du diagramme de diffraction neutronique du composé amorphe $\mathrm{Y}_{7} \mathrm{Fe}_{3}$ au cours de sa cristallisation [Kilcoyne et al. 2001], mesures effectuées sur une source continue grâce à l'instrument D20 de l'ILL [Convert et al. 1998].

\section{IV.b.2. Etude de transition de phases structurales}

La méthode des poudres offre l'avantage d'obtenir rapidement un diagramme de diffraction et permet de suivre les changements de symétrie si l'on fait varier les conditions expérimentales (température, pression). La thermodiffraction est un outil important pour le cristallographe car elle permet de déterminer les diagrammes de phases. Dans l'exemple du composé TiNi [Fruchart et al 93] représenté figure 7, la structure cristalline passe successivement par trois symétries différentes : cubique (austénite), hexagonale, puis monoclinique (martensite) lorsque la température diminue. Alors que la structure cubique présente à $320 \mathrm{~K}$ est caractérisée 
par peu de réflexions de Bragg dans le domaine angulaire étudié (18-98 20), de nouveaux pics de diffraction apparaissent lors du refroidissement, attestant de l'abaissement de la symétrie cristalline. Signalons que ces transitions sont d'origine displacive et que ce composé est un alliage dit «à mémoire de forme» et présente des propriétés intéressantes pour des applications technologiques, tant pour ses propriétés mécaniques que pour son potentiel à stocker l'hydrogène.

L'étude neutronique a démontré que, contrairement aux premières interprétations, l'état intermédiaire n'est pas une phase incommensurable mais correspond à un arrangement hexagonal.

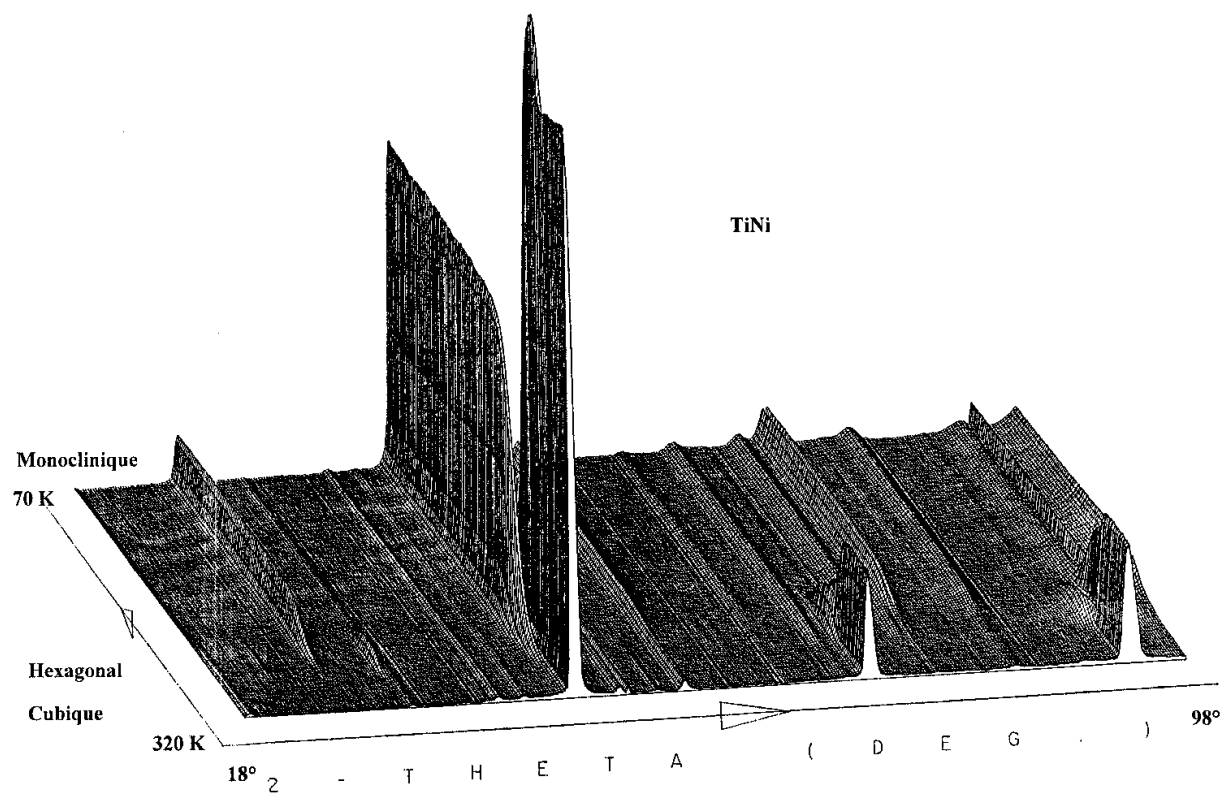

Figure 7: Mise en évidence de transformations structurales par thermodiffraction neutronique sur poudre du composé TiNi. Expérience effectuée sur l'instrument DIB de l'I.L.L. [Fruchart et al. 1993]

Un autre exemple est l'étude de la transition structurale du composé $\mathrm{NaNiO}_{2}$ qui, comme l'indique la figure 8 , présente un changement d'ordre cristallin passant du groupe d'espace $\mathrm{C} 2 / \mathrm{m}$ à température ambiante à une structure rhomboédrique R-3m au dessus de $480 \mathrm{~K}$. Une étude récente [Chappel 2000] a permis de relier cette transition structurale à la mise en ordre orbitale. Au dessus de $480 \mathrm{~K}$, l'ordre orbital disparaît. Ce système, monoclinique à température ambiante, subit une transition structurale vers $480 \mathrm{~K}$ et devient rhomboédrique. Cette transition, qui a une largeur de $20 \mathrm{~K}$ pendant laquelle les deux phases coexistent, est déplacée de $35 \mathrm{~K}$ selon que l'échantillon est chauffé ou refroidi. La dégénérescence orbitale des niveaux $\mathrm{e}_{g} \mathrm{du} \mathrm{\textrm {Ni } ^ { 3 + }}$ est levée par un effet JahnTeller coopératif, l'élongation des octaèdres $\mathrm{NiO}_{6}$ favorisant l'occupation de l'état orbital $\left|3 \mathrm{z}^{2}-\mathrm{r}^{2}\right\rangle$. La transition structurale présente, comme la susceptibilité magnétique, une très forte hystérèse autour de la transition Jahn-Teller. La comparaison des données structurales (par des mesures de diffraction de neutrons sur poudre) et des mesures magnétiques a conduit à une analyse détaillée de l'effet Jahn-Teller coopératif dans $\mathrm{NaNiO}_{2}$. En outre, un affinement de Rietveld des diagrammes enregistrés permet aussi de connaître les changements des positions atomiques (voir le chapitre décrivant la technique de Rietveld). A titre d'exemple, les affinements de Rietveld des deux formes structurales sont présentés sur la figure 9. La structure basse température est monoclinique et peut être déduite de la structure rhomboédrique haute température en considérant que les ions d'oxygène forment désormais un arrangement hexagonal distordu. Les octaèdres 
$\mathrm{NiO}_{6}$ (et $\mathrm{NaO}_{6}$ ) sont distordus (allongés) avec 4 distances $\mathrm{Ni}-\mathrm{O}$ courtes de $1,91 \AA$ et deux longues de $2,41 \AA$ à $300 \mathrm{~K}$, tandis qu'à haute température il n'y a qu'une distance Ni-O de $1,986 \AA$. Cette distorsion est caractéristique des ions Jahn-Teller tels que le $\mathrm{Ni}^{3+}$ dans la configuration bas spin $\mathrm{t}^{6}{ }_{2 \mathrm{~g}} \mathrm{e}_{\mathrm{g}} \mathrm{g}$. Tous les octaèdres $\mathrm{NiO}_{6}$ subissent la même élongation et une distorsion macroscopique est ainsi observée dans $\mathrm{NaNiO}_{2}$. Cette transition structurale s'accompagne de modification des propriétés magnétiques dont la compréhension n'a été possible que grâce à l'étude structurale.

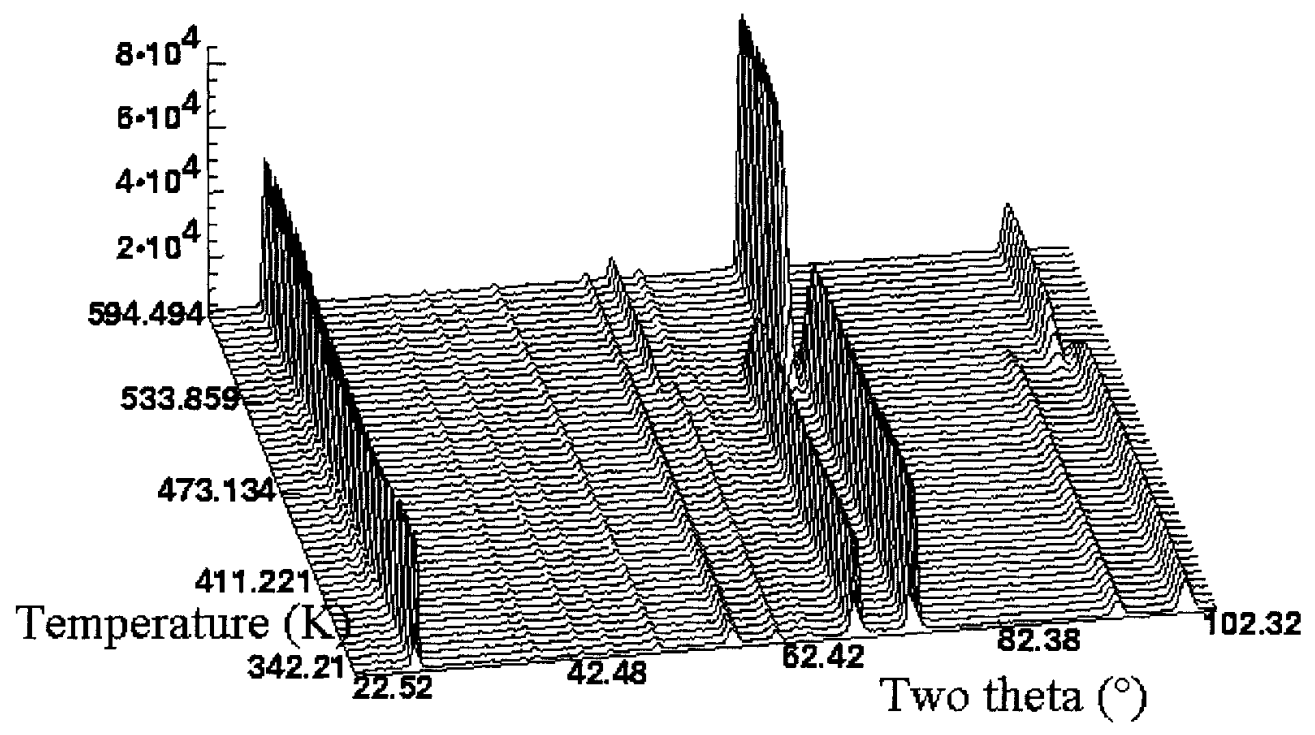

Figure 8 : Evolution thermique du diagramme de diffraction neutronique sur poudre de $\mathrm{NaNiO}_{2}$ [Chappel et al. 2000]
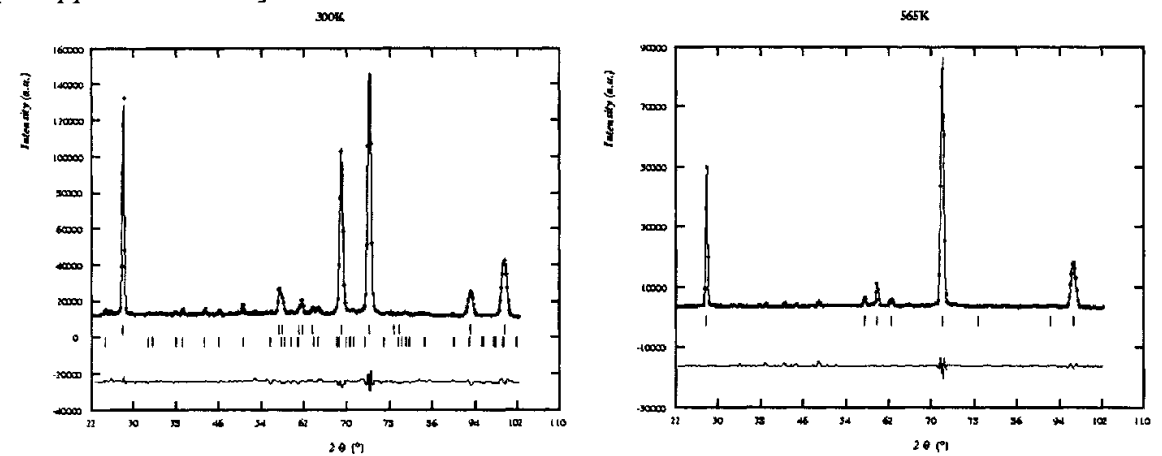

Figure 9: Comparaison des diagrammes de diffraction de neutrons sur poudre de $\mathrm{NaNiO}_{2}$ obtenus à $300 \mathrm{~K}$ (gauche) et $565 \mathrm{~K}$ (à droite). Les figures comparent les diagrammes calculés par la méthode de Rietveld (ligne continue) aux points expérimentaux. La courbe différence est tracée en bas du diagramme[Chappel et al. 2000]

\section{IV.b.3 Etude de transition de phase magnétique}

Une utilisation désormais classique de la diffraction neutronique consiste à déterminer le diagramme de phase magnétique de nouveaux matériaux par thermodiffraction. L'apport des neutrons est ici déterminant car leur extrême sensibilité au magnétisme de la matière permet d'avoir des pics de diffraction magnétique dont l'intensité peut être de l'ordre ou supérieure à celle 
des pics d'origine nucléaire. C'est le cas pour le composé MnRhAs dont l'évolution thermique du diagramme de diffraction de poudre est présentée sur la figure 10 . Le cas du pic $(0,0,1 / 4)$ est à cet égard significatif : il résulte uniquement de la diffraction magnétique des neutrons et possède à basse température une intensité largement supérieure à celle des pics de structure nucléaire (100), (001) et (110). Sans rentrer dans le détail du diagramme de phase magnétique de ce composé qui peut être trouvé en référence [Chenevier 1990], il est clair que au dessus de $161 \mathrm{~K}$ il existe un ordre antiferromagnétique qui conduit à une maille magnétique double par rapport à la maille nucléaire. Comme l'atteste la présence d'un pic de type $(0,0,1 / 2)$, ce doublement s'effectue selon l'axe c de la maille cristalline. Puis après un régime intermédiaire caractérisé par une structure complexe, un nouvel ordre s'établit à basse température, caractérisé par l'apparition entre autre du pic $(0,0,1 / 4)$. A titre d'information, une introduction à la diffusion magnétique des neutrons peut être trouvée dans les références suivantes [Brianso et Isnard 2000, Schweitzer 1994] ainsi que dans les cours de l'école thématique de l'an dernier .

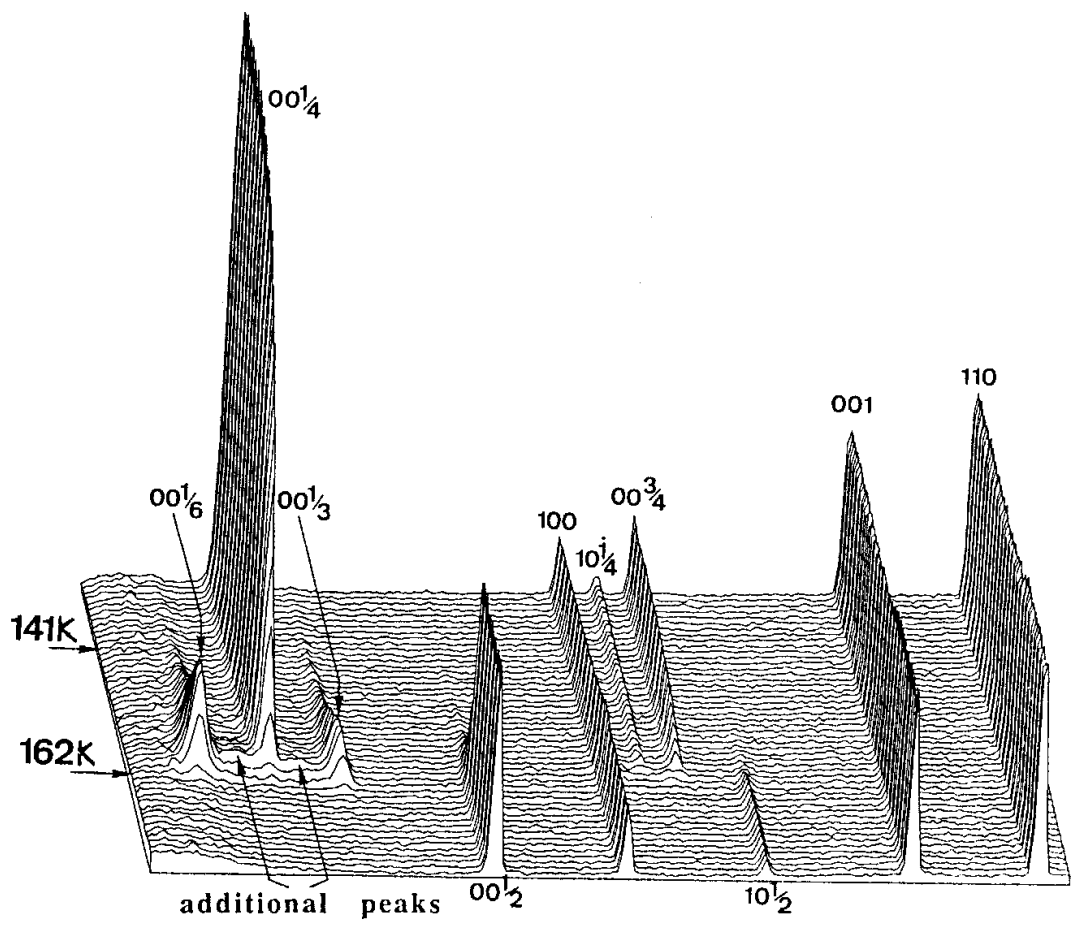

$2 \theta$

Figure $10:$ Evolution du diagramme de diffraction neutronique sur poudre du composé MnRhAs en fonction de la température et de l'angle $2 \theta$. Instrument DIB à l'I.L.L. L'indexation des pics est donnée dans la maille nucléaire.[Chenevier 1990].

Signalons que ce type d'étude peut être complété, voire validé, par l'utilisation de techniques complémentaires telles que la diffraction neutronique (polarisée ou non) sur monocristaux [Chenevier 1990]. 
IV.c. Etudes en temps réel et in situ de réactions chimiques

\section{IV.c.1. Etude de réaction solide-gaz}

La diffraction neutronique est un outil de choix pour les analyses réalisées in situ. Un exemple est donné sur la figure 11 qui représente l'évolution du diagramme de diffraction neutronique au cours de la réaction solide-gaz entre l'alliage $\mathrm{NdFe}_{11} \mathrm{Ti}$ et l'azote gazeux selon la réaction suivante :

$$
2 \mathrm{NdFe}_{11} \mathrm{Ti}+\mathrm{x} \mathrm{N}_{2}=>2 \mathrm{NdFe}_{11} \mathrm{TiN}_{\mathrm{x}} .
$$

On observe successivement la nitruration de l'alliage sous forme de $\mathrm{NdFe}_{11} \mathrm{TiN}_{\mathrm{x}}$, ce qui se traduit sur le diagramme par un décalage des pics de diffraction vers les petits angles. Ce décalage de la position des pics de Bragg résulte de l'expansion du réseau cristallin qui se produit lorsque les atomes d'azote s'insèrent dans le réseau cristallin. Puis, à plus haute température, la décomposition du matériau est attestée par la disparition des pics de Bragg du composé $\mathrm{NdFe}_{11} \mathrm{TiN}_{\mathrm{x}}$ et l'apparition progressive d'un seul pic intense caractéristique du fer sous forme alpha. Ces expériences réalisées sur 1'instrument D20 de 1'ILL ont permis de déterminer le domaine de température dans lequel la réaction d'insertion de l'azote peut être réalisée mais aussi de mettre en évidence le faible domaine de stabilité thermique de la phase $\mathrm{NdFe}_{11} \mathrm{TiN}_{\mathrm{x}}$. Cette phase se décompose très tôt en donnant un nitrure de néodyme amorphe (invisible sur le diagramme présenté ici) de type $\mathrm{NdN}$ et du fer $\alpha$ dont on voit ici le pic (110). Les indices de Miller des pics de Bragg sont donnés à titre indicatif sur la figure 11. Ces résultats et en particulier la métastabilité de la phase $\mathrm{NdFe}_{11} \mathrm{TiN}_{\mathrm{x}}$ ont été déterminants pour exclure cette phase, aux propriétés magnétiques pourtant prometteuses, de la course aux matériaux pour aimants hautes performances [Oleinek 2000, Oleinek et al 2000].

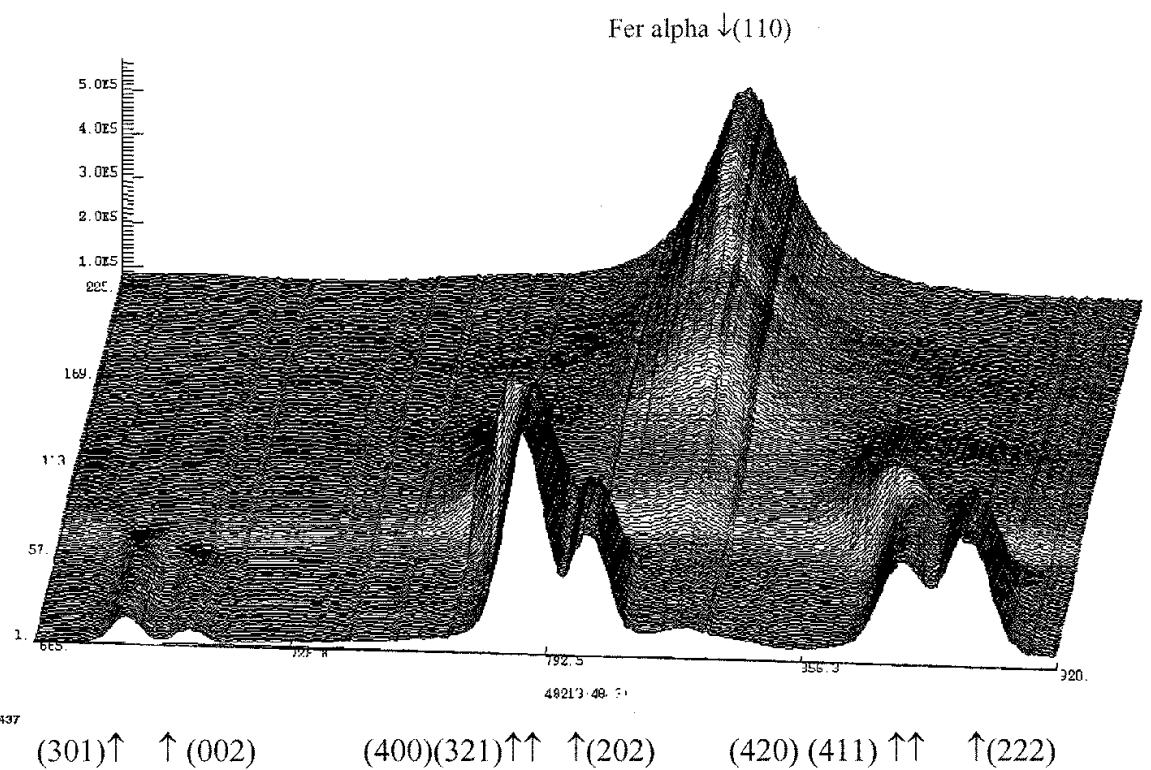

Figure 11 : Etude de réaction chimique réalisée "in situ" sous faisceau de neutron entre un composé de $\mathrm{NdFe}_{11} \mathrm{Ti}$ et l'azote gazeux [Oleinek et al 2000]. Mesures effectuées sur l'instrument D20 de l'ILL. 
Outre l'évolution de l'intensité d'un pic de diffraction, il est aussi souvent intéressant d'analyser la forme des pics de diffraction, elle peut renseigner sur l'état de cristallisation du composé, sa microstructure (taille, forme des cristallites...), la présence ou non de contraintes dans le matériau, les directions et intensités de ces contraintes.

Ainsi, l'observation de la figure 11 révèle que le pic du fer est nettement plus large que la résolution instrumentale, ce qui indique que les particules de fer ainsi formées à basse température sont mal cristallisées. Il est alors possible de déterminer la taille des cristallites et de suivre leur croissance au cours du processus.

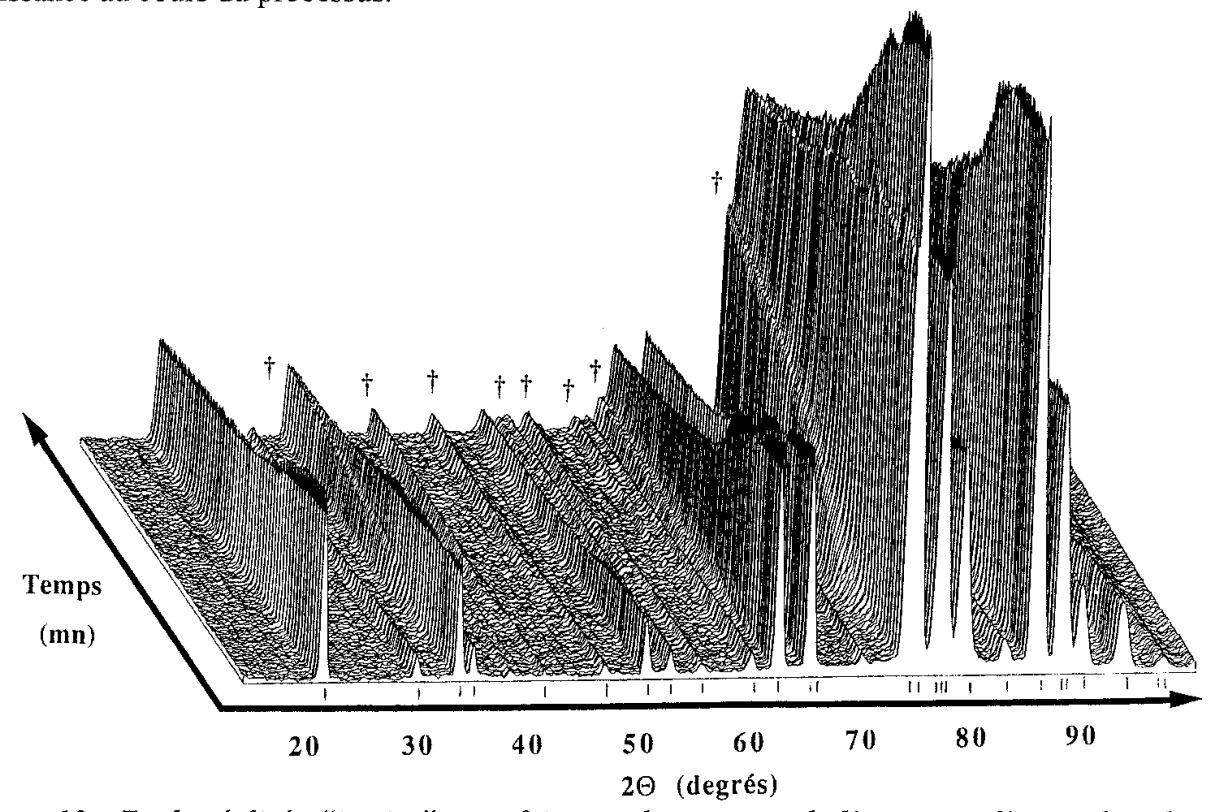

Figure 12 : Etude réalisée "in situ" sous faisceau de neutrons de l'insertion d'azote dans la structure du composé $\mathrm{Nd}_{2} \mathrm{Fe}_{17}$.[Isnard et al 1992a].

Une étude équivalente avait été menée précédemment sur l'instrument D1B [Isnard et al. 1992a] pour étudier l'insertion de l'azote dans la structure du composé $\mathrm{Nd}_{2} \mathrm{Fe}_{17}$. Même si c'est la phase $\mathrm{Sm}_{2} \mathrm{Fe}_{17} \mathrm{~N}_{3}$ qui présente les meilleures caractéristiques en terme d'applications pour aimant permanent, le samarium naturel présentant un forte absorption des neutrons thermiques, l'étude de la nitruration s'est naturellement reportée sur le composé isotype au néodyme. Pour optimiser les conditions de synthèse de ces nouveaux matériaux et aussi pour comprendre comment se passe l'insertion de l'azote dans la structure, la diffraction neutronique fut un outil précieux. Ainsi l'échantillon de poudre de $\mathrm{Nd}_{2} \mathrm{Fe}_{17}$ a été mis en réaction avec l'azote gazeux dans un four porté à $450^{\circ} \mathrm{C}$. L'enregistrement séquentiel des diffractogrammes de neutrons est représenté sur la figure 12. Les positions des pics de Bragg de la phase $\mathrm{Nd}_{2} \mathrm{Fe}_{17}$ sont indiquées par des traits le long de l'axe des abscisses tandis que les positions des pics de Bragg correspondant à la phase $\mathrm{Nd}_{2} \mathrm{Fe}_{17} \mathrm{~N}_{3}$ sont indiquées par les signes suivants : $\uparrow$. On observe une croissance progressive des pics de Bragg correspondant à la phase $\mathrm{Nd}_{2} \mathrm{Fe}_{17} \mathrm{~N}_{3}$. Cette étude a démontré que contrairement à l'hydrogène qui peut occuper deux sites interstitiels différents [Isnard et al. 1992b], l'un octaédrique et l'autre tétraédrique, l'azote, plus gros, ne s'insère que dans les sites octaédriques de la structure. Ces sites interstitiels sont situés dans l'environnement proche des atomes $\mathrm{Nd}$. La structure cristallographique correspondante, représentée sur la figure 13, indique les différents sites octaédriques et tétraédriques notés D1 et D2 respectivement. Cela a permis d'établir que la 
teneur maximale en azote est alors de 3 atomes par formule $\mathrm{Nd}_{2} \mathrm{Fe}_{17}$ et non de 6 ou 8 comme il avait été envisagé auparavant. Enfin, le suivi précis de la position des réflexions de $\mathrm{Nd}_{2} \mathrm{Fe}_{17} \mathrm{~N}_{3}$ ainsi que les affinements de Rietveld ont démontré que l'insertion de l'azote se fait par la coexistence de deux phases, l'une $\mathrm{Nd}_{2} \mathrm{Fe}_{17}$ et l'autre $\mathrm{Nd}_{2} \mathrm{Fe}_{17} \mathrm{~N}_{3}$. Enfin, entre autres résultats, l'existence d'une solution solide de type $\mathrm{Nd}_{2} \mathrm{Fe}_{17} \mathrm{Nx}$ avec $0<\mathrm{x}<3$ a pu être écartée. Les fractions volumiques normalisées de chaque phase $\mathrm{Nd}_{2} \mathrm{Fe}_{17}$ et $\mathrm{Nd}_{2} \mathrm{Fe}_{17} \mathrm{~N}_{3}$ ont pu être déduites de l'affinement de profil du diagramme de diffraction biphasique ( $\mathrm{cf}$ figure 12 ). Il est alors possible d'en déduire les caractéristiques de la cinétique de réaction elle même.

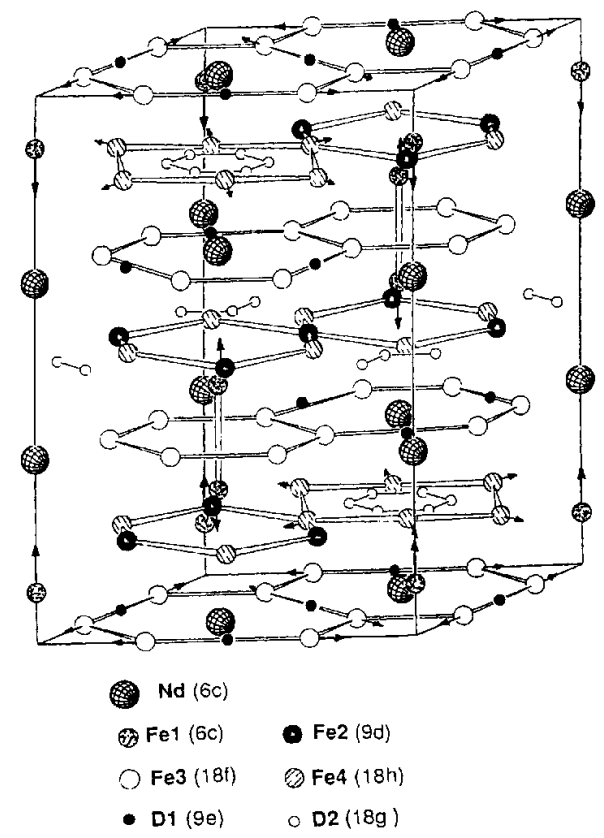

Figure 13 : Structure cristallographique du composé $\mathrm{Nd}_{2} \mathrm{Fe}_{17}$ présentant deux sites interstitiels, l'un octaédrique (D1) et l'autre tétraédrique (D2).[Isnard et al. 1992]

Dans le cas de l'insertion de l'hydrogène dans le composé composé $\mathrm{Nd}_{2} \mathrm{Fe}_{17}$, il a été possible de déterminer la localisation de l'hydrogène mais également de suivre en temps réel sa désorption. Cette étude [Isnard et al. 1992] a été menée avec le deutérium plutôt que l'hydrogène afin de diminuer la diffusion incohérente. La figure 14 indique clairement l'évolution du taux d'occupation des deux sites interstitiels, D1 (octaédrique) et D2 (tétraédrique), au cours de la réaction de désorption. Cela a permis de mettre en évidence la plus grande stabilité thermique du site octaédrique, qui conserve les atomes de deutérium jusqu'à une température $T_{2}$ approchant les $500 \mathrm{~K}$, alors que à cette température les sites tétraédriques sont vides. Une étude structurale fine à en outre permis de comprendre comment la présence de deutérium modifie la structure cristallographique en provoquant un réarrangement atomique complet schématisé par les flèches noires sur la figure 13. Comme l'illustre la partie droite de la figure 14, l'évolution des distances inter-atomiques les plus courtes $(\mathrm{Fe} 1-\mathrm{Fe} 1)$ est directement reliée à la présence ou non d'atomes interstitiels sur les sites octaédriques. Ces distances $\mathrm{Fe}-\mathrm{Fe}$ sont très importantes du point de vue des interactions d'échanges et conditionnent les propriétés physiques. Le suivi in situ a permis de mieux comprendre les propriétés magnétiques de ces matériaux. 

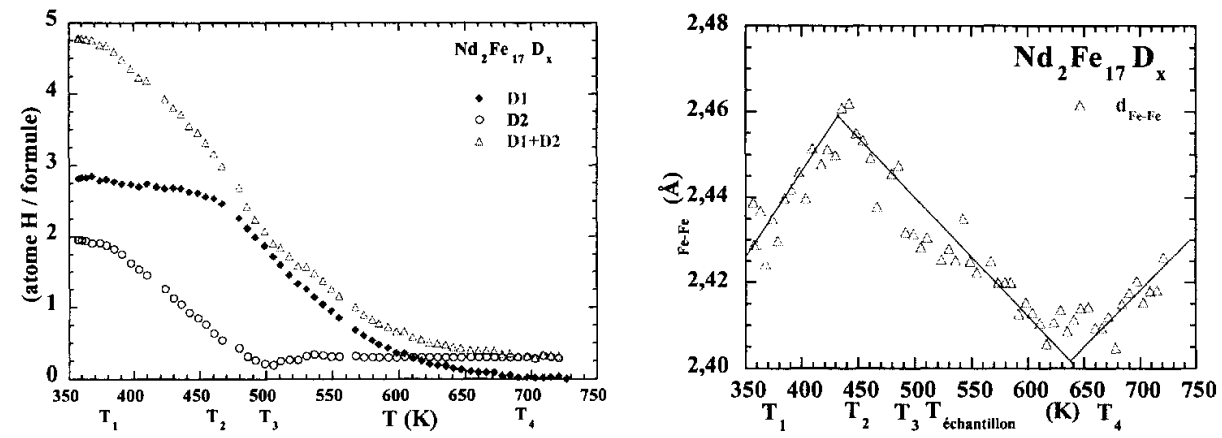

Figure 14 : Evolution thermique de la teneur en deutérium dans le composé $\mathrm{Nd}_{2} \mathrm{Fe}_{I 7} \mathrm{D}_{x}$ (à gauche) et conséquences sur les distances interatomiques les plus courtes (à droite) [Isnard et al. 92].

\section{IV.c.2. Analyse électrochimique in situ}

La diffraction de neutrons in situ a été développée très tôt sur les sources à haut flux tel que l'I.L.L,. en particulier pour l'électrochimie. En électrochimie, il convient de signaler quelques difficultés expérimentales: les matériaux constituant la cellule électrochimique (électrode, électrolyte, enceinte...) ne doivent pas être trop absorbants ni donner lieu à des pics de diffraction qui risqueraient de masquer la diffraction de l'échantillon étudié. Ces contraintes ne sont cependant pas incontournables, en particulier avec les neutrons qui présentent la spécificité d'être très peu absorbés par nombre de matériaux. Ainsi la diffraction de neutrons in situ a été développée très tôt sur les sources à haut flux tel que l'I.L.L. Signalons aussi que lorsque le milieu électrolyte est protoné, cela peut donner lieu à de la diffusion incohérente. Cette difficulté peut être aisément contournée soit en travaillant en milieu deutéré plutôt qu'en milieu protoné, soit en enregistrant le diagramme de diffraction en l'absence de l'électrolyte. Dans le second cas, l'enregistrement du diagramme est alors fait pendant une interruption du courant en l'absence d'électrolyte.

De nombreuses études structurales ont pu être réalisées avec succès tant sur le système $\mathrm{H} / \gamma-\mathrm{MnO}_{2}$ des piles Leclanché [Chabre 93, Chabre 1995, Rippert 1991, Rippert 1990] que sur le comportement d'hydrures intermétalliques de terres-rares, matériaux qui sont utilisés comme électrode négative des batteries $\mathrm{NiMH}_{\mathrm{x}}$ [Latroche et al 1999]. Une description détaillée des cellules électrochimiques développées pour la diffusion neutronique peut être trouvée en référence [Latroche et al 2000 et Chabre 2001].

Signalons que, même s'il est aussi possible d'effectuer des études in situ par diffraction de rayons X [Notten 1994] sur ces systèmes, l'apport des neutrons a été déterminant pour aboutir à une meilleure compréhension des mécanismes mis en jeu au cours des charges et décharges des piles et en particulier pour étudier les paramètres limitant le nombre de recharges possibles de ces accumulateurs. Les hydrures métalliques dérivant de la structure $\mathrm{LaNi}_{5}$ sont largement étudiés pour leur capacité de stockage de l'hydrogène. Ils sont utilisés industriellement pour la production des électrodes négatives de piles rechargeables nickel-hydrure métallique ( dites piles Ni-MH). Ces batteries possèdent des capacités de stockage $20 \%$ supérieures à celles des piles nickelcadnium. La substitution du nickel par le cobalt offre le double intérêt d'améliorer la résistance à la corrosion et de prolonger la durée de vie des piles rechargeables en permettant un plus grand nombre de cycles charge-décharge. La réaction à l'électrode négative consiste à insérer de l'hydrogène dans le réseau cristallin de manière réversible afin de pouvoir charger et décharger la pile. 
L'électrode négative est le siège de la réaction suivante : $R M_{5} \Rightarrow R M_{5} H_{x}$ où $R$ représente le lanthane ou un de ses substituants (éléments de terre rare essentiellement) et $\mathrm{M}$ représente un élément de transition (principalement un élément 3d). Dans les piles rechargeables $\mathrm{Ni}-\mathrm{MH}_{\mathrm{x}}$, l'électrode positive est quant à elle constituée d'hydroxydes de nickel de taille nanométrique. Des études neutroniques [Chabre et al. 2001] sont actuellement en cours sur l'instrument D1B pour caractériser l'évolution structurale de cet hydroxyde de nickel lors du cyclage de l'électrode positive.

L'étude en temps réel et in situ par diffraction neutronique a permis de démontrer que la plus grande cyclabilité des électrodes riches en cobalt est reliée à la présence d'une phase intermédiaire dite $\gamma$ [Latroche et al. 1999]. Une étude récente a démontré que ce mécanisme est aussi à la base de la longévité plus grande des piles sur-stoechiométriques en métal de transition comportant une faible teneur en cobalt [Latroche et al. 2001]. La figure 15 montre une partie du diagramme de diffraction neutronique obtenu sur une cellule électrochimique lors de la charge de l'électrode. On observe nettement la disparition progressive des pics de diffraction correspondant à l'intermétallique, c'est-à-dire à la phase $\alpha$. Il y a aussi une intensification progressive des pics, liée à l'hydrure phase $\beta$. L'insertion de l'hydrogène conduit à une augmentation importante de la maille cristalline et les pics de diffraction apparaissent donc à plus bas angle que ceux de la phase $\alpha$. Une phase transitoire de type $\gamma$ apparaît, caractérisée par la présence d'un pic sur la droite de la figure 15. Il est notable que la position des pics de Bragg des phases $\gamma$ et $\beta$ est pratiquement constante au cours de la réaction, montrant que les volumes des mailles sont quasiment constants. En revanche, les raies correspondant à la phase $\alpha$ évoluent en position ce qui atteste d'une teneur en hydrogène variable au cours du processus. Durant les cinq premières heures de charge, le comportement classique à deux phases $\alpha$ et $\beta$ est observé, les proportions de phase $\alpha$ et $\beta$ variant de façon opposée ( $\alpha$ diminue tandis que $\beta$ augmente). Puis une troisième phase $\gamma$ correspondant à un hydrure intermédiaire apparaît, sa quantité atteint progressivement $45 \%$ au bout de 12 heures de charge puis diminue au profit de la phase $\beta$ pour n'être plus que de $20 \%$ à la fin de la charge (et $70 \%$ de phase $\beta$ ). Le comportement de l'électrode lors de la décharge est notablement différent puisque très rapidement la maille de la phase $\beta$ évolue et tend vers celle de la phase $\gamma$. En conclusion, la meilleure stabilité au cyclage électrochimique des composés étudiés a été attribuée à la présence de cette phase métastable qui n'est présente que lors de la charge. La présence de cette phase réduit de manière notable l'expansion volumique provoquée par les fortes contraintes mécaniques qui accompagnent l'insertion d'hydrogène et limite ainsi la pulvérisation du matériau lors des charges et décharges successives. Limitant la mise en poudre de l'alliage, cela favorise aussi la résistance à la corrosion de l'électrode.

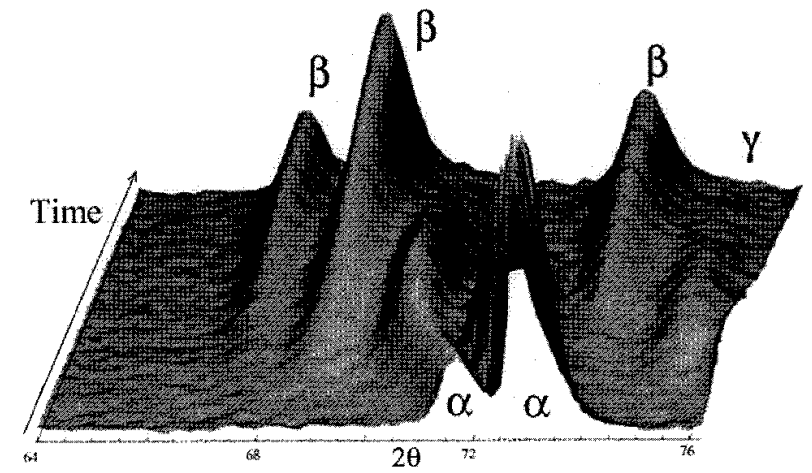

Figure 15 : Représentation en trois dimensions de l'évolution temporelle d'une partie du diagramme de diffraction neutronique lors de la charge d'une électrode positive de batterie NiMH [Latroche et al. 2001]. 
Ce type d'étude électrochimique convient bien à la diffusion neutronique car sur des instruments tels que D1B ou D20 (à l'Institut Laue Langevin), les temps d'acquisition par diagramme de diffraction sont de l'ordre de la minute ce qui reste relativement court par rapport aux temps caractéristiques des batteries. Il est alors possible de suivre la dynamique structurale associée aux perturbations électrochimiques lors des charges et décharges des batteries. En outre, le suivi en parallèle des données électrochimiques ( potentiel, courant, charge...) fournissent des données complémentaires qui, confrontées à l'analyse des paramètre structuraux, viennent enrichir les résultats obtenus. On peut aussi signaler que des études par diffraction de rayons $\mathrm{X}$ peuvent aussi être menées in situ en électrochimie, soit à partir de source classique [Notten 1994], soit à partir de rayonnement synchrotron.

\section{Intercalation of $\mathrm{THF}$ in $\mathrm{CsC}_{24}$}
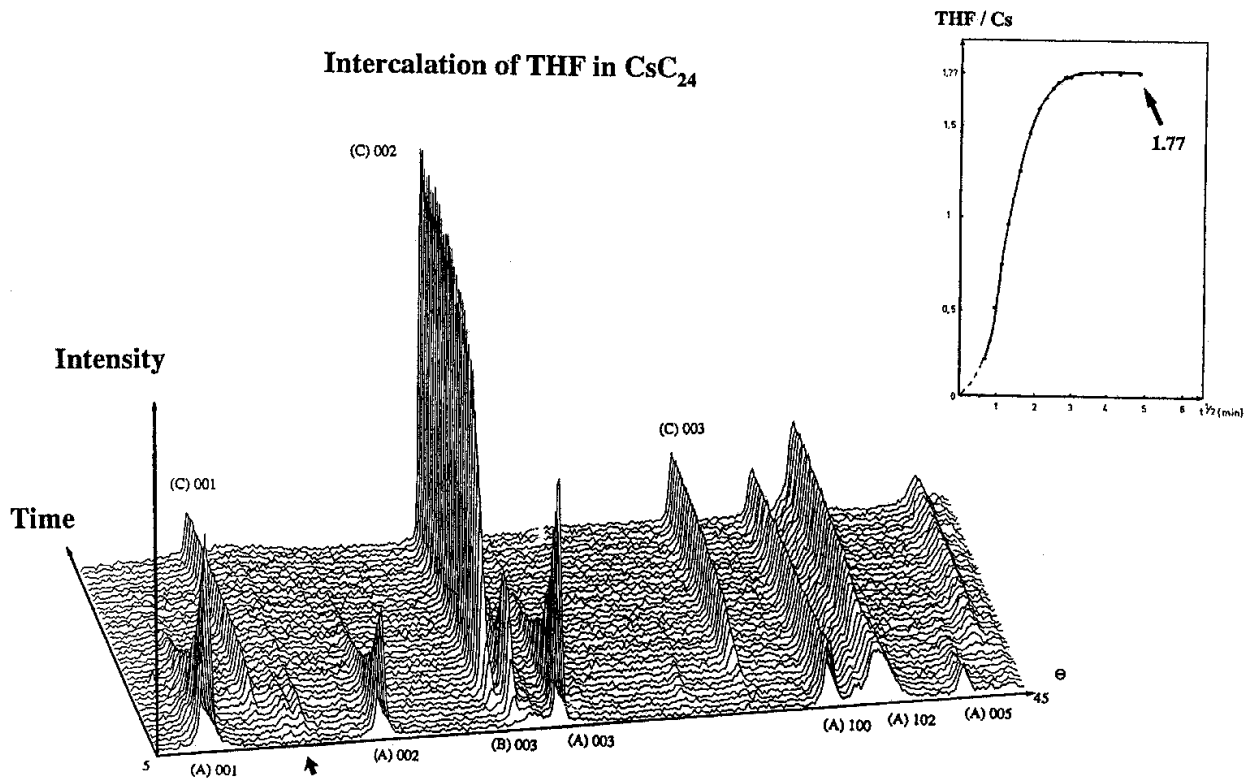

Figure 16: Evolution des diagrammes de diffraction durant l'intercalation du tétrahydrofurane (THF) dans CsC 24 [Goldmann et al. 1998 et 1989].

\section{IV.c.3. Réaction d'intercalations chimiques dans le graphite}

La diffraction neutronique a été utilisée avec succès pour caractériser l'intercalation de diverses espèces dans le graphite dont des alcanes [Goldmann et al. 1998 et 1989]. En raison du caractère lamellaire du graphite, l'intercalation consiste en l'insertion de l'espèce chimique entre les plans de graphène. Selon les conditions d'intercalation et la nature de l'espèce intercalée, différents stades d'intercalation peuvent se présenter, c'est-à-dire différentes phases ordonnées. Ces ordres peuvent être définis par la séquence périodique de couches intercalées et de couche de graphène. La valeur du stade $\mathrm{s}$ indique le nombre de plans de graphène séparant deux couches intercalées. De nombreuses expériences de ce type ont été menées sur D1B par l'équipe du Professeur Béguin. La figure 16 indique l'évolution temporelle du diagramme de diffraction neutronique obtenu lors de la réaction de la vapeur de tétrahydrofurane (THF) sur le deuxième stade d'intercalation de $\mathrm{CsC}_{24}$. Cette réaction dure quelques heures à température ambiante et a été 
réalisée avec du THF deutéré. Pour analyser en détail les différents stades mis en jeu, un temps d'acquisition d'une minute fut choisi. L'étude a démontré que la transformation structurale se passe en trois étapes : la phase binaire de graphitures de césium $\mathrm{CsC}_{24}$ disparaît rapidement alors qu'une phase intermédiaire identifiée comme caractéristique d'un stade 2 de type $\mathrm{CsC}_{24}(\mathrm{THF})_{\mathrm{x} \approx 1}$ apparaît. Quelques minutes plus tard, l'étape finale correspondant à un stade 1 apparaît sur le diagramme de diffraction, le diagramme étant caractéristique de la phase ternaire $\mathrm{CsC}_{24}$ $(\mathrm{THF})_{x \approx 1,7}$. Neuf minutes après le départ de la réaction, l'intensité des pics correspondant à la phase intermédiaire passe par un maximum avant de décroître. Après cinq heures, le composé $\mathrm{CsC}_{24}$ a disparu complètement. Une phase intermédiaire comme celle observée ici a une durée de vie limitée, elle serait passée inaperçue si la fréquence d'acquisition avait été inférieure. Or, la connaissance de son existence permet de mieux comprendre les mécanismes mis en jeu lors de l'intercalation. Il est donc important de définir le temps d'acquisition le plus petit possible. En effet, même lorsque l'on effectue une étude in situ et en temps réel, il est aussi possible de passer à côté de certains phénomènes à cause de conditions expérimentales insuffisantes ou mal définies.

Dans ce type d'étude, il est possible de suivre la cinétique d'intercalation. On peut donc ici tirer le meilleur parti de la résolution temporelle de l'acquisition. Cela a été mis à profit par H. Pillière dans sa thèse [Pillière 91]. Il a ainsi effectué de nombreuses intercalations d'alcanes dans $\mathrm{CsC}_{36}$ à différentes températures. A titre d'exemple, l'évolution temporelle du diagramme de diffraction neutronique durant l'intercalation à $327 \mathrm{~K}$ du n-pentane dans $\mathrm{CsC}_{36}$ est présentée sur la figure 17 , tandis que les aspects de cinétique sont représentés sur la figure 18.

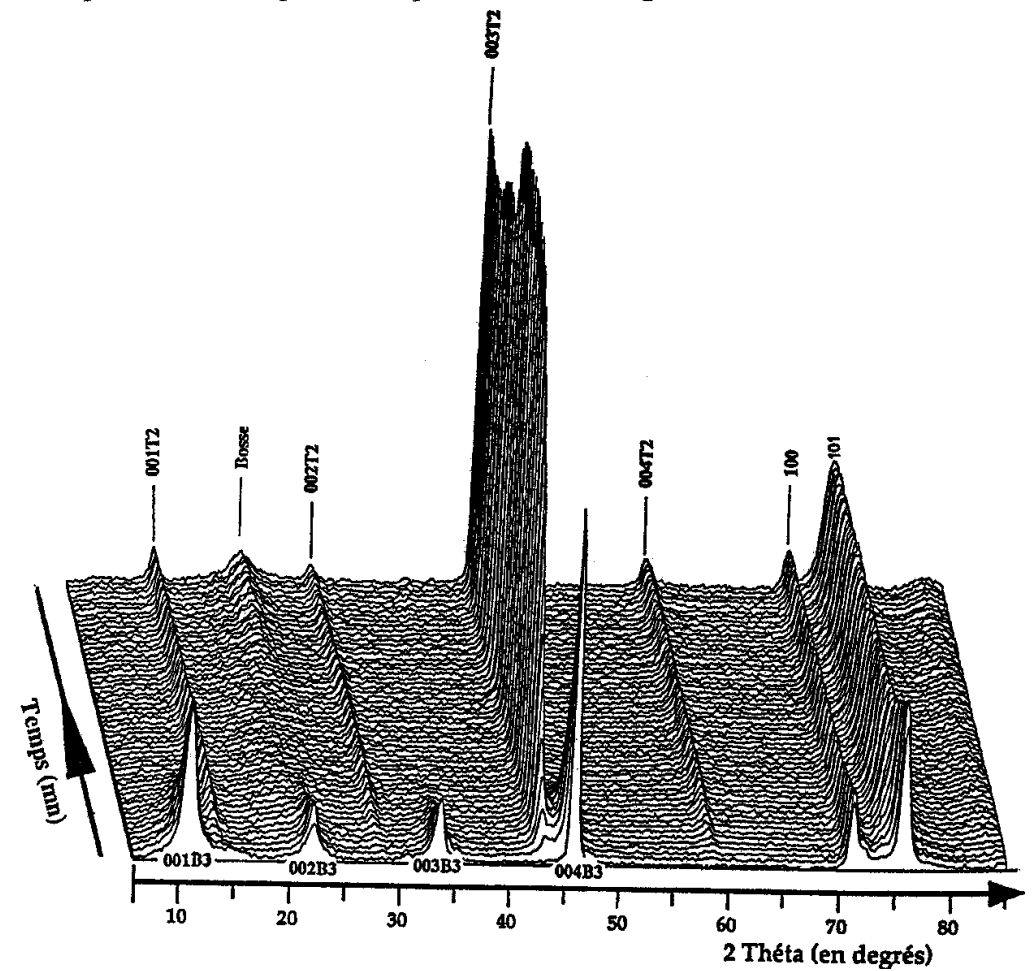

Figure 17: Evolution des diagrammes de diffraction durant l'intercalation du n-pentane dans $\mathrm{CsC}_{36}$ [ Pillière 91]. 


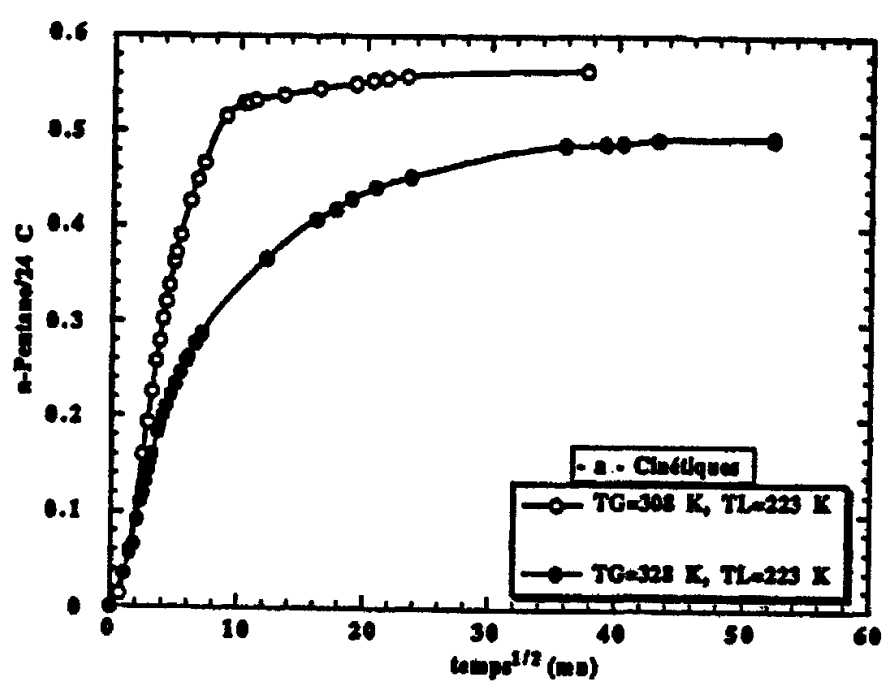

Figure 18: Courbes représentant la cinétique d'intercalation isotherme du n-pentane dans $C_{s} C_{36}$ pour une température du graphite de 308 et $328 \mathrm{~K}$ correspondant aux symboles o et • respectivement [Pillière 1991].

\section{IV.c.4. Déshydratation}

La diffraction des neutrons sur poudre permet aussi de suivre des réactions de déshydratation telle que la déshydratation d'un hydrate d'oxyde de molybdène (dit aussi acides molybdiques). L'analyse de la déshydratation par calorimétrie différentielle (figure 19) montre deux pics endothermiques, les études thermogravimétriques ont, elles aussi, révélé deux étapes lors de la déshydratation.
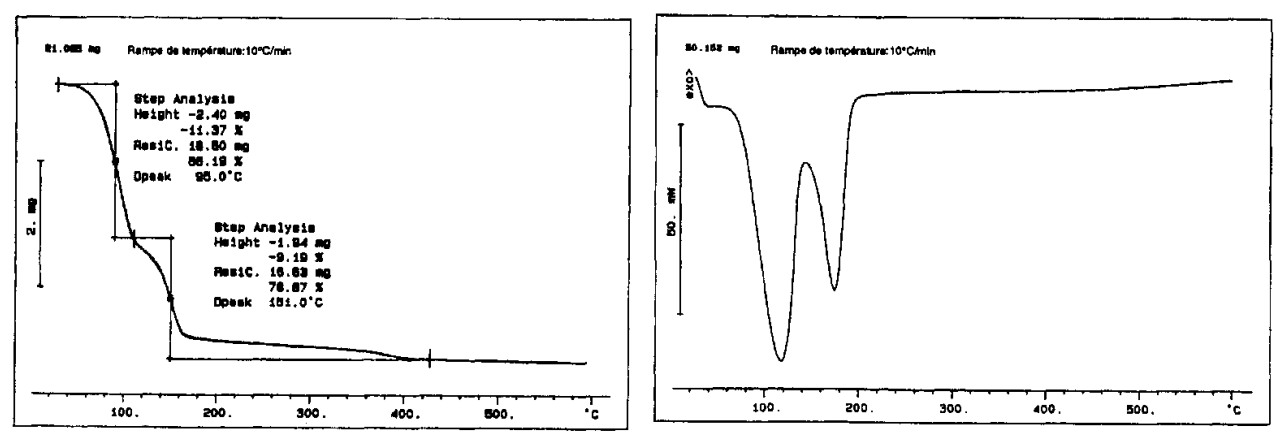

Figure 19 : Evolution des signaux thermogravimétriquse (gauche) et de DSC (droite) obtenus lors de la déhydratation de la phase $\mathrm{MoO}_{3} .2 \mathrm{H}_{2} \mathrm{O}$

Ainsi l'étude neutronique [Boudjada et al. 1993, Anne et al. 1990] menée en temps réel a permis de suivre les mécanismes topotactiques mis en jeu au cours de la déshydratation provoquée par le l'augmentation progressive de la température ambiante jusqu'à $400^{\circ} \mathrm{C}$ avec une vitesse de rampe de $20^{\circ} / \mathrm{h}$. Les diagrammes de diffraction ont été enregistrés toutes les 3 minutes. A partir d'une analyse structurale multi-phases, il a été possible de suivre en température la proportion de 
chacune des phases en présence. Le diagramme de diffraction neutronique est présenté sur la figure 20. On observe une forte évolution du diagramme de diffraction neutronique au cours du chauffage. La structure du composé $\mathrm{MoO}_{3} .2 \mathrm{H}_{2} \mathrm{O}$ consiste en un assemblage d'octaèdres distordus de type $\mathrm{MoO}_{5}\left(\mathrm{H}_{2} \mathrm{O}\right)$ connectés par les sommets, formant ainsi des feuillets. Ces feuillets s'empilent le long de la direction [010]. La deuxième molécule d'eau se trouve alors sous forme inter-lamellaire entre les feuillets, la cohésion de la structure étant assurée par des liaisons hydrogène.

L'étude neutronique réalisée sur D1B a montré que le processus de déshydratation se produit en deux étapes majeures. Ceci est clairement visible sur le diagramme $3 \mathrm{D}$ de la figure 20 Les molécules d'eau quittent la structure en deux étapes principales, passant de $\mathrm{MoO}_{3}, 2 \mathrm{H}_{2} \mathrm{O}$ (monoclinique) à $\mathrm{MoO}_{3}, \mathrm{H}_{2} \mathrm{O}$ puis à $\mathrm{MoO}_{3}$ (orthorhombique). Cela se traduit en particulier par une décroissance du bruit de fond lorsque les molécules d'eau quittent la structure, puisque l'hydrogène possède une forte contribution à la diffusion incohérente. Le domaine de stabilité du monohydrate a pu être estimé à environ $30^{\circ} \mathrm{C}$. Cependant, une analyse plus détaillée des résultats par affinement de chaque diagramme de diffraction permet de suivre l'évolution des paramètres de maille au cours de la déshydratation. Ainsi, la détermination de l'évolution thermique des paramètres de maille pour chaque phase a mis en évidence une anomalie de dilatation à $100^{\circ} \mathrm{C}$ et révélé l'existence d'une nouvelle transition de phase. Vers $100^{\circ} \mathrm{C}$, le monohydrate $\mathrm{MoO}_{3}, \mathrm{H}_{2} \mathrm{O}$ subit un réarrangement. Deux formes cristallines différentes de monohydrate $\mathrm{MoO}_{3}, \mathrm{H}_{2} \mathrm{O}$ sont successivement observées selon la température. La déshydratation doit être décrite selon le processus suivant :

$$
\mathrm{MoO}_{3}, 2 \mathrm{H}_{2} \mathrm{O} \Rightarrow>\mathrm{MoO}_{3}, \mathrm{H}_{2} \mathrm{O}(\mathrm{I})=>\mathrm{MoO}_{3}, \mathrm{H}_{2} \mathrm{O}(\mathrm{II}) \Rightarrow \mathrm{MoO}_{3}
$$

L'évolution de la taille des cristallites de phase $\mathrm{MoO}_{3}$ a pu être déterminée à partir de l'analyse de l'élargissement des pics de diffraction de cette phase et la direction [021]* a été identifiée comme un axe de croissance privilégié lors de la formation de la phase $\mathrm{MoO}_{3}$. Plus de détails peuvent être trouvés dans les références suivantes [Boudjada et al. 1993, Anne et al. 1990, Seguin 1994].

\section{Thermal dehydration of $\mathrm{MoO}_{3}, 2 \mathrm{H}_{2} \mathrm{O}$}

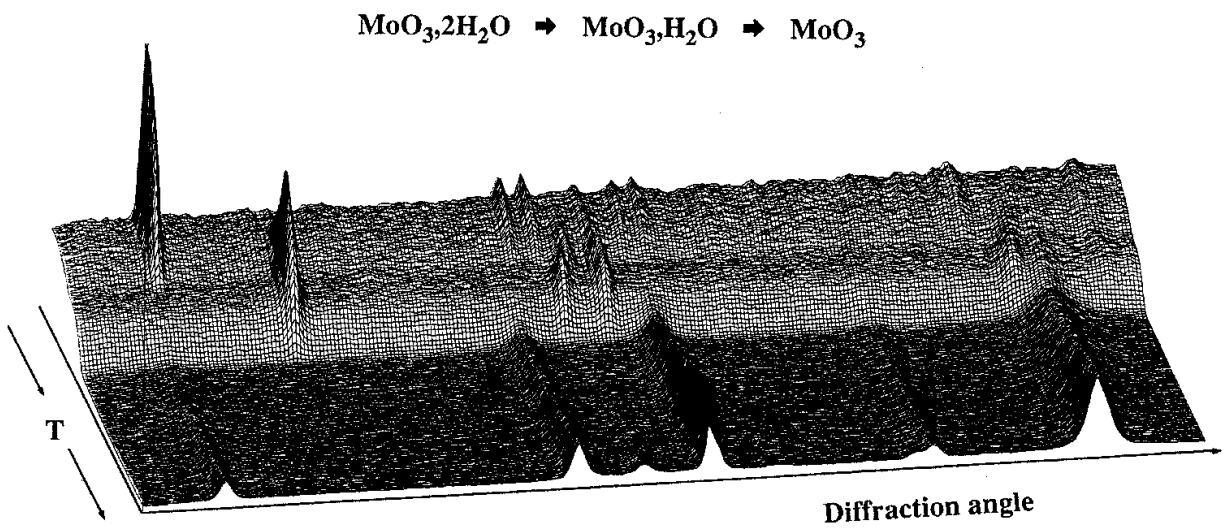

Figure 20 : Evolution thermique du diffractogramme lors du chauffage de $\mathrm{MoO}_{3} .2 \mathrm{H}_{2} \mathrm{O}$, conduisant à $\mathrm{MoO}_{3} . \mathrm{H}_{2} \mathrm{O}$ puis à $\mathrm{MoO}_{3}$. 


\section{IV.c.5. Synthèse de composé sous flux de neutrons}

La synthèse de quasicristaux est délicate pour plusieurs raisons. Elle repose souvent sur la connaissance de diagramme de phase complexe souvent ternaire ou quaternaire. Cette synthèse nécessite des températures élevées. Dans leur ouvrage intitulé "Les quasicristaux matière à paradoxes $\gg[$ Janot et Dubois 1998], Janot et Dubois montrent bien l'apport déterminant de la diffraction neutronique sur la progression des connaissances des phases quasicristallines. Lors de l'étude d'alliage de composition $\mathrm{Al}_{65} \mathrm{Cu}_{20} \mathrm{Fe}_{15}$, la diffraction neutronique a révélé de manière directe les transformations de phase qui produisent le quasicristal. Initialement à l'état liquide, l'alliage a été progressivement refroidi de $1325 \mathrm{~K}$ jusqu'à $1040 \mathrm{~K}$, température ou s'achève la formation du solide. Depuis l'état liquide, quatre étapes de formations de phase solide se succèdent. Chacune peut aisément être identifiée par la présence de pics de Bragg sur la figure 21. La phase icosaédrique ne se forme donc pas à partir de l'alliage $\mathrm{Al}_{65} \mathrm{Cu}_{20} \mathrm{Fe}_{15}$ liquide mais plutôt à la suite d'une série de réactions entre liquides et cristaux solides. Les quasicristaux se forment à la périphérie de phases solides cristallines qui les précèdent.

La thermodiffraction a donc été riche d'enseignement sur le diagramme de ces phases quasicristallines, mais si l'on reste à température constante en suivant l'évolution temporelle du diagramme de diffraction neutronique, il est possible de sonder la cinétique de croissance du quasicristal. Il a pu être montré que la formation de la phase quasicristalline est parfaitement réversible. L'intensité des pics de Bragg est directement proportionnelle à l'augmentation de la fraction volumique de la phase en cours de croissance. Il est donc facile de suivre la cinétique de croissance et d'en déduire les mécanismes qui la régissent :

- réaction contrôlée par la germination aux joints de grains sans diffusion à longue distance,

- réaction contrôlée par la diffusion en volume,

- cela a été réalisée par diffraction neutronique [Janot et Dubois 1998] sur différents systèmes icosaédriques: $\mathrm{AlCuFe}, \mathrm{AlPdMn}$, lequels ne présentent pas les mêmes mécanismes de croissance.

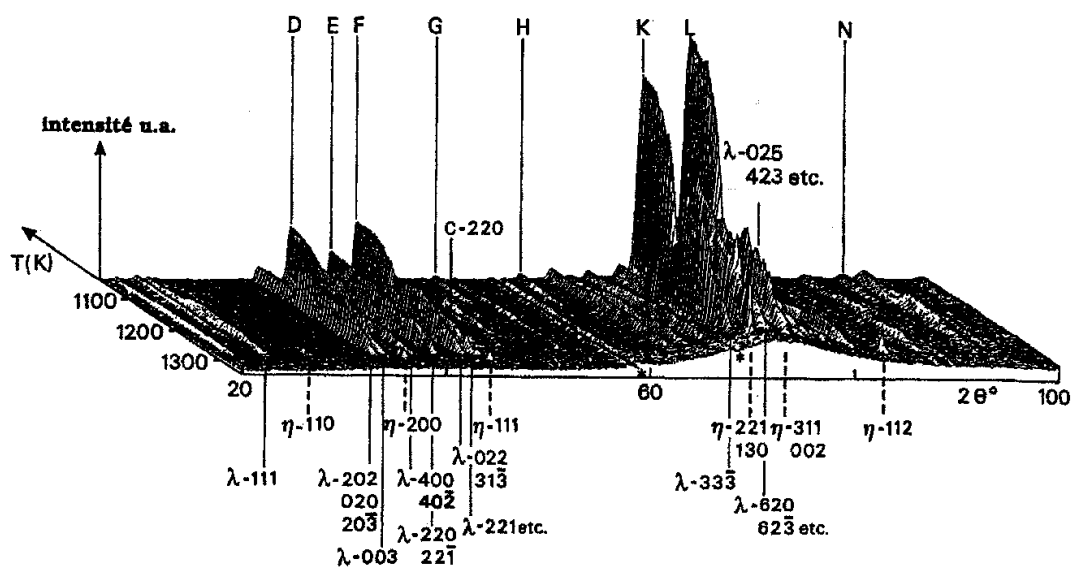

Figure 21: Etude par diffraction neutronique en temps réel de la formation de la phase icosaédrique dans un alliage $\mathrm{Al}_{65} \mathrm{Cu}_{20} \mathrm{Fe}_{15}$ dont les réflexions sont indiquées par des lettres. Les autres repères et indices de Miller caractérisent les phases intermédiaires [Chuang-Dong et al. 1989]. 
Synthèse d'hexaferrite : l'hexaferrite de baryum dite hexaferrite $M$ est un composé de structure hexagonale de formule chimique $\mathrm{BaFe}_{12} \mathrm{O}_{19}$. Le suivi in situ [Menarde et al. 1989] de la synthèse de ce composé par réaction de $\gamma \mathrm{Fe}_{2} \mathrm{O}_{3}$ et d'une phase amorphe riche en baryum a pu être réalisé par diffusion de neutrons sur D1B (cf figure 22). Cette seule étude neutronique a permis de déterminer les étapes de la réaction de synthèse, la vitesse de croissance mais aussi l'anisotropie de forme des cristallites de $\mathrm{BaFe}_{12} \mathrm{O}_{19}$. C'est l'évolution en température de la largeur des raies de Bragg qui a permis d'estimer les tailles apparentes des cristallites le long des différentes directions cristallographiques. Il ressort clairement que les particules se développent sous forme de plaquettes et que leur croissance s'effectue essentiellement perpendiculairement à l'axe [001] comme l'indique la figure 22 .
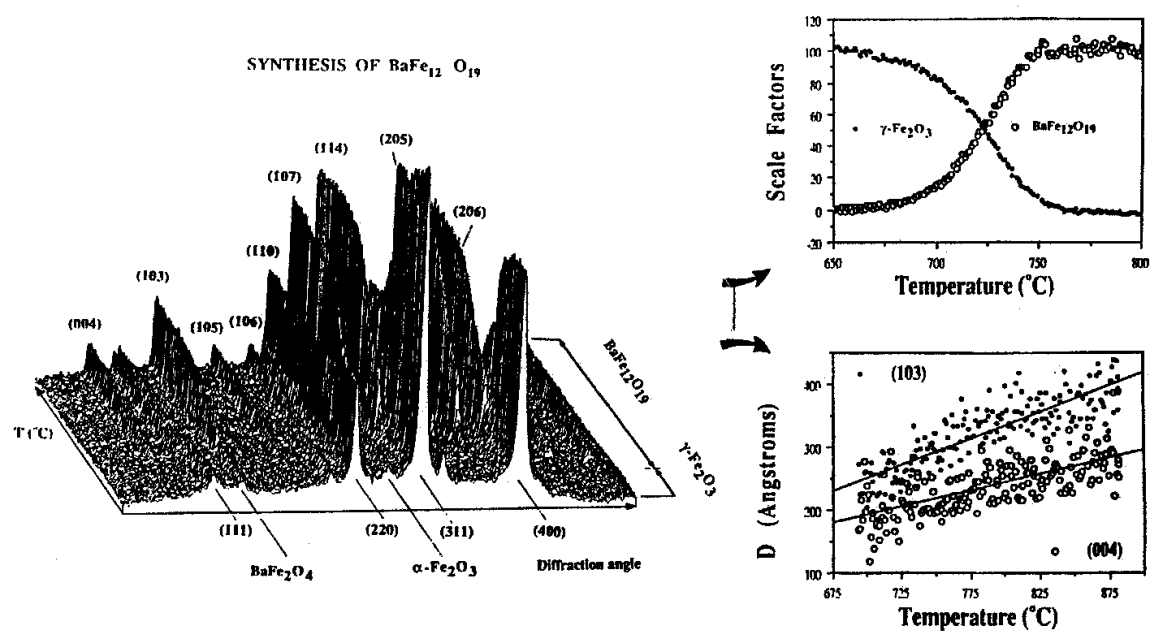

Figure 22: Evolution thermique des diagrammes de diffraction de neutrons au cours de la réaction entre une phase amorphe riche en $\mathrm{Ba}$ et $\gamma \mathrm{Fe} 2 \mathrm{O} 3$ pour former l'hexaferrite $\mathrm{BaFe}_{12} \mathrm{O}_{19}$ [Menarde et al. 1989].

De nombreuses études neutroniques se sont penchées sur les cuprates supraconducteurs à haute température de transition afin de mieux comprendre leur structure et leurs propriétés physiques. Dans ces matériaux, la température critique est fortement dépendante de la teneur en oxygène. Une étude de l'oxydation de $\mathrm{YBa}_{2} \mathrm{Cu}_{3} \mathrm{O}_{6}$ en $\mathrm{YBa}_{2} \mathrm{Cu}_{3} \mathrm{O}_{7}$ a été réalisée par diffraction en temps réel sur l'instrument D1B de l'I.L.L. [Rodriguez et al. 1988]. Dans cette étude par diffraction des neutrons sur poudre, effectuée sous oxygène pur, un diagramme de diffraction a été enregistré toutes les 1,5 minutes, soit au total 900 diagrammes collectés durant un cycle de montée en température et descente ( voir figure 23 ). Les analyses effectuées par affinement de profil ont démontré la forte discontinuité des paramètres de mailles observée à la transition quadratiqueorthorombique entre 260 et $335^{\circ} \mathrm{C}$. En revanche, la transition inverse vers $660^{\circ} \mathrm{C}$ est presque continue. Lors de l'oxydation, la transition structurale est du premier ordre avec coexistence de deux phases sur environ $75^{\circ} \mathrm{C}$. Signalons aussi qu'une très faible augmentation de la composition en oxygène est suffisante pour initier le changement de symétrie. Enfin, comme l'indique la figure 23, la quantité d'oxygène sur chacun des sites cristallographiques a pu être étudiée. 


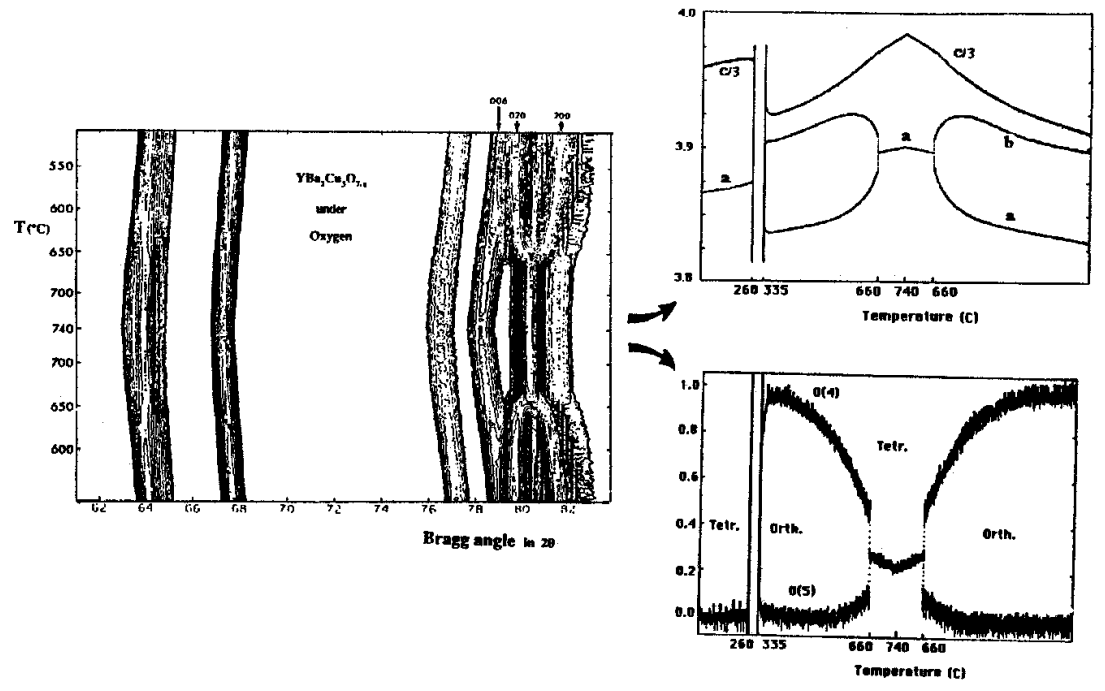

Figure 23 : Evolution du diagramme de diffraction neutronique du composé $\mathrm{YBa}_{2} \mathrm{Cu}_{3} \mathrm{O}_{7-x} . \mathrm{La}$ partie gauche représente un tracé de contour permettant de suivre les modifications structurales. L'évolution des paramètres de mailles est représentée en haut à droite, tandis que les fractions d'occupation des sites d'oxygène $O(4)$ et $O(5)$ sont données en bas à droite.

IV.d. Informations structurales contenues en dehors des pics de Bragg :

La diffraction est un outil d'investigation très riche mais l'information structurale contenue dans un diagramme de diffraction ne se retrouve cependant pas uniquement dans les pics de diffraction. Ainsi, l'évolution du bruit de fond du diagramme peut être riche d'enseignement sur l'évolution du processus réactionnel. Nous avons noté, sur la figure 20, que le bruit de fond n'est pas constant mais qu'il présente une nette décroissance lorsque la température augmente. Cela traduit directement la perte en hydrogène du composé de départ. En effet, l'hydrogène possède vis-à-vis des neutrons une section efficace de diffusion incohérente qui est 45 fois plus élevée que celle de diffusion cohérente. Sa présence dans le matériau (ici sous forme de molécule d'eau) se traduit donc par une forte augmentation du niveau de bruit de fond. Le suivi de l'amplitude du bruit de fond peut donc ici être utilisé pour mesurer la teneur en hydrogène du matériau.

Dans certains composés, une forte ondulation du bruit de fond peut être observée : c'est par exemple le cas de divers conducteurs ioniques comme la phase $\mathrm{La}_{1.7} \mathrm{Bi}_{0.3} \mathrm{Mo}_{2} \mathrm{O}_{9}$ dont le diagramme de diffraction neutronique est représentée sur la figure 24 [ Goutenoire et al. 2001]. La présence de bosse de diffusion diffuse dans le bruit de fond a été discutée par Guinier dans son ouvrage de référence [Guinier 1964]. Il est possible dans cet exemple de tirer une information structurale quantitative de ce comportement spécifique du fond continu à partir de la formule de Debye [Debye 1915] qui exprime la contribution d'un désordre structural statique à la diffusion diffuse élastique. Dans le cas de la phase $\mathrm{La}_{1.7} \mathrm{Bi}_{0.3} \mathrm{Mo}_{2} \mathrm{O}_{9}$ [Goutenoire et al 2001], cela traduit l'existence d'ordre à courte distance caractérisé par des paires oxygène-oxygène de l'ordre de $2,5 \AA$. Sans chercher à être exhaustif, citons que la présence de ce type de diffusion diffuse a aussi été observée en référence [Vannier et al. 1999]. 


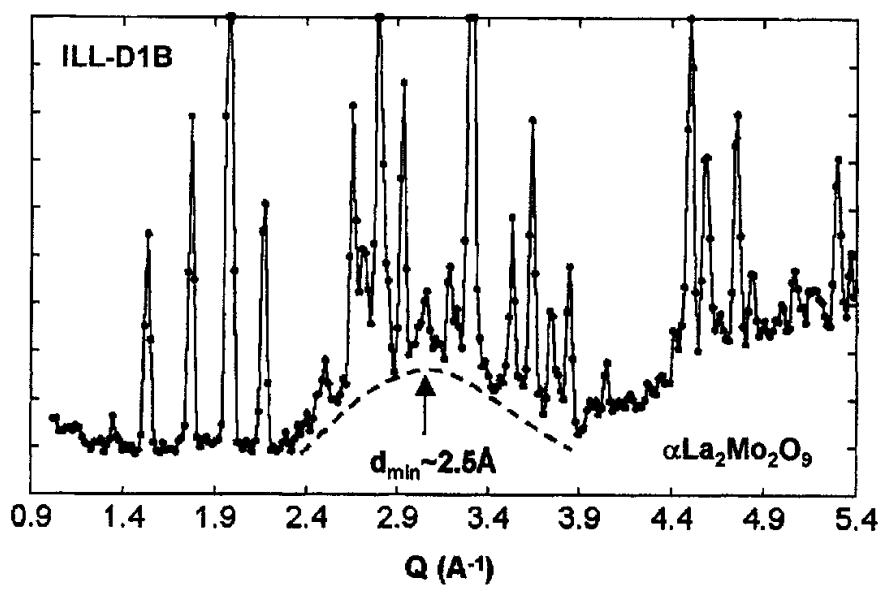

Figure 24 : Diagramme de diffraction neutronique obtenu à température ambiante sur le composé $L \mathrm{a}_{2} \mathrm{Mo}_{2} \mathrm{O}_{9}$, montrant un large pic de diffusion $\grave{a} Q=4 \pi \sin \theta / \lambda=3.1 A^{-1}$, dî̀ à un ordre à courte distance.

Comme l'illustrent ces quelques exemples, il est donc souvent riche d'enseignement d'analyser attentivement l'évolution du bruit de fond d'un diagramme de diffraction, cela peut compléter les informations issues de l'analyse des pics de diffraction.

\section{IV.e. Analyse des données de diffraction résolue dans le temps}

Le traitement des données de diffusion résolue en temps comporte une difficulté supplémentaire par rapport aux études statiques. En effet, ce type d'expériences génère un grand nombre de diagrammes, cela peut aller jusqu'à plusieurs centaines voire quelques milliers. Il est donc inenvisageable de faire un dépouillement un à un de ces diagrammes. Dans le cas de la diffraction de poudre, l'utilisation de programme d'affinement Rietveld qui permet un affinement cyclique est alors nécessaire. Le principe consiste à enchaîner les affinements de profil de telle sorte que le résultat d'affinement du diagramme $\mathrm{n}$ est utilisé comme point de départ pour l'affinement du diagramme $\mathrm{n}+1$. Parmi ces programmes, le programme Fullprof développé par J. Rodriguez Carvajal [Rodriguez 93] à l'ILL puis au LLB est très bien adapté à ces analyses en temps réel.

Le meilleur guide pour l'analyse d'expériences réalisées in situ est sans conteste le tracé et l'étude de diagramme 3D ou de tracés de contour qui permettent de visualiser l'évolution de la diffusion de neutrons au cours du processus suivi. A titre d'exemple, un diagramme de contour qui représente en fait des courbes d'iso-intensité d'un diagramme de diffraction neutronique est présenté sur la figure 25 . Ce tracé de contour correspond en fait à une autre représentation de la figure 11. Le domaine en température dans lequel se passe la transition de phase ainsi que les évolutions des positions des pics de Bragg sont plus facilement identifiables sur un tracé de contour. Il faut alors traquer tout changement d'intensité, de position, toute apparition ou disparition de pics bref tous les indices de changement ou d'évolution. Cela permet ensuite de cerner les différents stades de l'évolution du diagramme et d'adapter sa stratégie d'affinement et d'analyse au problème rencontré. En raison de la faible statistique de collecte ou du domaine angulaire restreint accessible, il n'est pas toujours possible d'affiner tous les paramètres d'un problème donné. L'observation du digramme $3 \mathrm{D}$ et 1 'affinement des diagrammes extrêmes 
premiers et derniers de la séquence permettent en général de cerner les paramètres pertinents. Il est aussi possible de s'appuyer sur des informations complémentaires ( pression, température, potentiel électrochimique, masse de l'échantillon etc...) pour limiter le nombre de paramètres significatifs... Cette sélection étant faite, il faut se lancer dans un affinement complet de la séquence de diagrammes enregistrés.

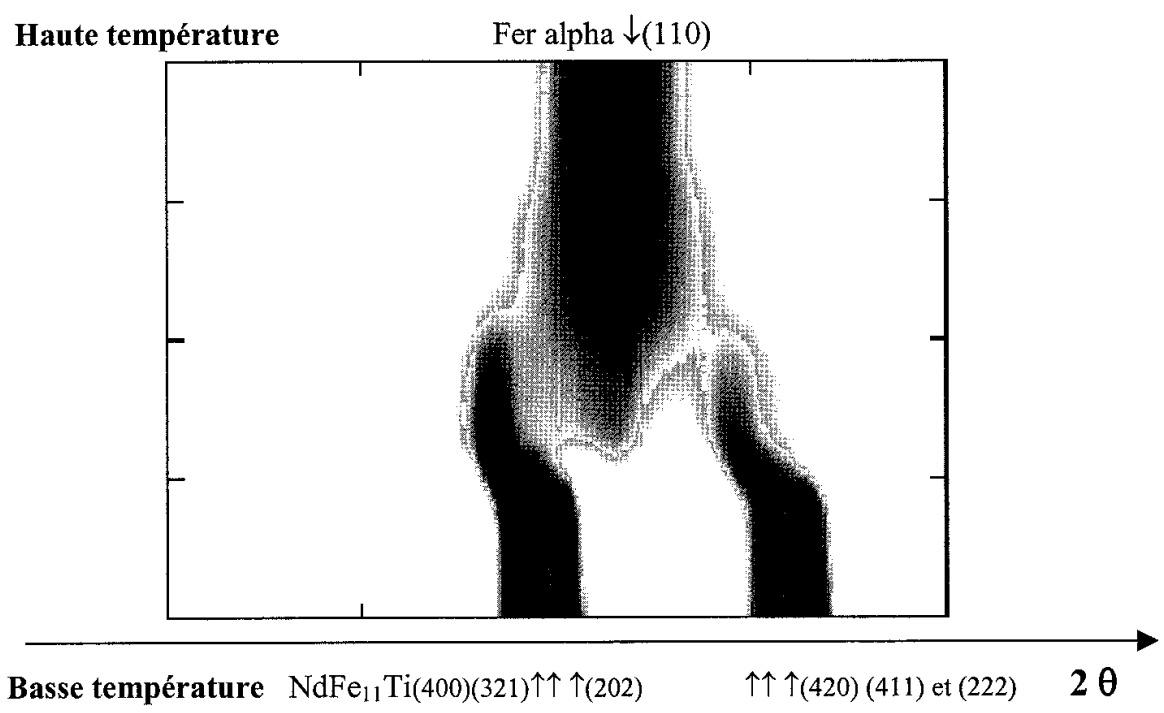

Figure 25: Tracé de contour d'isointensité correspondant à la réaction entre $\mathrm{NdFe}_{11} \mathrm{Ti}$ et l'azote gazeux [Oleinek et al. 2000,] mesures effectuées sur l'instrument D20 de l'ILL. Le décalage progressif des pics de Bragg correspond à l'insertion d'azote dans le réseau, puis à plus haute température, la phase se décompose en donnant du fer alpha.

\section{ANALYSES PAR DIFFUSION AUX PETITS ANGLES}

L'avantage de la diffusion aux petits angles est de renseigner sur des objets allant de quelques Angströms à environ $1000 \AA ̊$. Ces objets sondés peuvent être des hétérogénéités diverses: précipités, pores, cavités... Cette technique d'analyse est très utilisée, tant en diffusion de rayons $X$ qu'en diffusion neutronique. La diffusion aux petits angles de neutrons est une technique bien adaptée pour l'étude non destructive de la microstructure d'alliages. La diffusion de neutrons aux petits angles fournit des renseignements quantitatifs sur la distribution de taille, fraction volumique, le nombre de ces hétérogénéités.

Les superalliages à base de nickel sont constitués d'une phase $\gamma$ de structure cubique à face centrée à l'intérieur de laquelle précipite une phase ordonnée $\gamma^{\prime}$ de structure différente. L'existence de ces précipités est essentielle pour l'obtention de propriétés mécaniques optimales à haute température comme le nécessite leur emploi comme aubes de turbine. Les précipités servent d'ancrage pour les dislocations, cela conduit à un durcissement structural. Il est donc possible de caractériser ces précipités $\gamma^{\prime}$ par diffusion de neutrons aux petits angles afin d'en connaître la forme ainsi que d'en déterminer les dimensions et même l'arrangement spatial. Les figures de diffusion aux petits angles (voir figure 26) sont surtout sensibles à la forme des précipités et peuvent présenter des pics de corrélation qui sont caractéristiques des interférences entre les ondes diffusées aux petits angles par des précipités voisins. Il est alors possible de déduire une distance 
moyenne $\lambda$ inter précipités de la position $\mathrm{q}_{\max }$ des pics de corrélations dans le réseau réciproque $\left(\lambda=2 \pi / q_{\max }\right)$. Ce type d'étude a été réalisé avec succès par D. Bellet et collaborateurs [Bellet 1990, Bellet 1992]. La diffusion aux petits angles a aussi permis de d'observer l'évolution au cours du temps de la coalescence orientée d'échantillons prédéformés [Veron et Bastie 1997]. Il y a regroupement des précipités selon une symétrie d'ordre deux comme l'indique la partie droite de la figure 27. Il est aussi possible de suivre l'évolution du signal diffusé aux petits angles en fonction du recuit thermique [Bellet et al. 92]. De manière complémentaire, la diffraction des neutrons peut être utilisée pour mesurer in situ l'évolution de la fraction volumique des précipités de la phase $\gamma^{\prime}$. Ce résultat est présenté sur la partie gauche de la figure 27. Ces mesures ont été effectuées sur l'instrument D11 de l'ILL avec des temps d'acquisition de l'ordre de 300 secondes. Ce type d'étude est fréquemment effectué pour caractériser les aciers mais la diffusion aux petits angles est aussi une technique fort utile pour la caractérisation des polymères [Cotton et Nallet 1999].
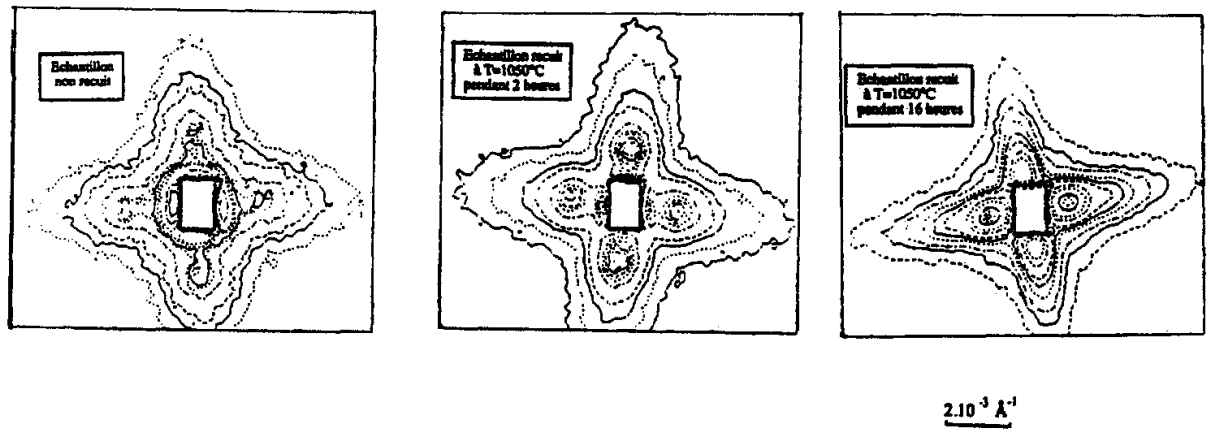

Figure 26: Courbe d'iso-intensité des figures de diffusion aux petits angles correspondant à un échantillon ayant subi différents traitements thermiques [Bellet 1997].

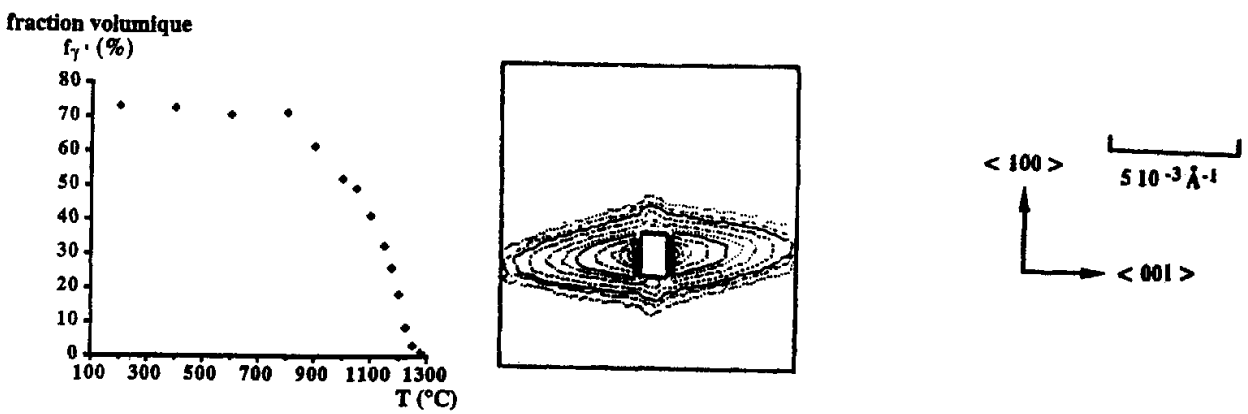

Figure 27 : Evolution thermique de la fraction volumique des précipités de phases $\gamma$ ' dans un superalliage (à gauche) [Bellet 1997]. Courbe d'iso-intensité des figures de diffusion aux petits angles correspondant $\grave{a}$ un échantillon ayant subi une déformation (à $T=1050^{\circ} \mathrm{C}$ avec $\sigma=140 \mathrm{MPa}$ ) [Veron et Basti 1997].

Les neutrons étant aussi sensibles au magnétisme de la matière, citons un travail original de Ishikawa et al. [Ishikawa ]. Ces auteurs ont utilisé la diffusion des neutrons aux petits angles pour étudier le processus de relaxation de l'aimantation dans un verre de spin à basse température sur une source pulsée.

Une équipe allemande a eu aussi souvent recours à la diffusion aux petits angles en temps réel [Eckold 1990, 1992, 2001] pour étudier la cinétique de décomposition dans les solides 
ioniques. La complémentarité de la diffusion aux petits angles avec la diffraction des neutrons est particulièrement bien utilisée dans l'article [Eckold 2001]. Quelques détails de ces travaux seront décrits dans le paragraphe traitant de la spectroscopie trois-axes résolue en temps.

Pour en savoir plus sur la diffusion des neutrons aux petits angles et ses applications, le lecteur peut se reporter au cours en français édité par Cotton et Nallet et publié chez EDP Sciences [Cotton et Nallet 1999] ou à l'article suivant : [Gerold et Kostorz 1978].

\section{SPECTROMETRIE TROIS AXES RESOLUE EN TEMPS}

La possibilité d'utiliser la spectroscopie neutronique trois axes en temps réel a été démontrée par G. Eckold [Eckold 1990]. En raison du flux relativement faible accessible par cette méthode, ce type d'expérience est limité en pratique aux processus réversibles que l'on peut reproduire de manière périodique ( $\mathrm{cf}$ partie III Méthodologie). L'acquisition de données doit être synchronisée avec le processus à étudier, puis la répétition de manière périodique de l'acquisition d'un diagramme de diffraction permet d'améliorer la statistique de comptage. L'avantage de la spectrométrie trois axes est ici de disposer d'un instrument sélectif dans l'espace Q- $\omega$ et d'une bonne résolution dans l'espace réciproque. Il est donc possible de tirer parti de ces avantages pour caractériser des phénomènes de physique comme de chimie, dont les temps caractéristiques peuvent s'étendre de $10^{-3}$ à $10^{4}$ secondes.

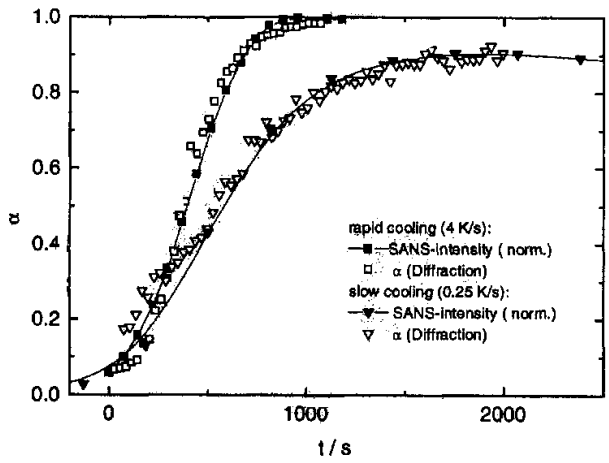

Figure 28 : Evolution temporelle de l'intensité du signal de diffusion de neutrons aux petits angles et du degré de décomposition de $\mathrm{Ag} 0,5 \mathrm{Na}{ }_{0,5} \mathrm{Br}$ à $470 \mathrm{~K}$ pour deux vitesses de refroidissement [Eckold 2001].

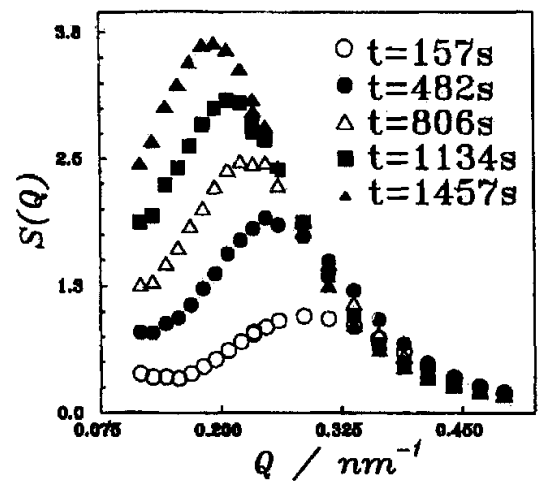

Figure 29: Evolution temporelle du signal de diffusion des neutrons aux petits angles lors d'une décomposition spinodale [Eckold 2001]. 
Un cas particulièrement intéressant concerne l'étude de la cinétique de décomposition spinodale de solide ionique $\mathrm{AgBr}-\mathrm{NaBr}$ [Eckold 2001]. Comme l'illustre les figures 28,29 et 30, G. Eckold a su mettre en œuvre deux techniques de diffusion de neutron en temps réel :

- la diffusion aux petits angles qui renseigne sur la taille des précipités,

- la diffraction qui permet de suivre l'évolution des paramètres de mailles.

L'originalité est aussi de pratiquer la diffraction en temps réel sur un instrument 3-axes. Cela a permis de mieux connâtre les mécanismes qui régissent la décomposition, d'isoler les régimes dominés par la nucléation croissance des régimes de fluctuation. Les deux techniques utilisées se sont avérées complémentaires puisque la diffraction n'a pas très été sensible à la cinétique de démixion dans le régime dit de fluctuation, en revanche, la diffusion aux petits angles des neutrons s'est avérée très précieuse dans ce domaine. Ainsi, la diffusion aux petits angles a permis de suivre l'évolution du volume des précipités au travers de la variation de la dépendance temporelle $\mathrm{Q}_{\max }$ et de l'intensité diffusée. L'investigation de ce phénomène rapide a pu être réalisée sur un réacteur de neutron ne disposant que d'un faible flux. Mais la faiblesse du flux incident a été largement compensée par l'ingéniosité des expérimentateurs qui ont su mettre en œuvre ces expériences de manière stroboscopique. Les informations recueillies peuvent l'être avec un pas de l'ordre de la seconde ou moins.

$$
\mathbf{x}=\mathbf{0 . 4 1}
$$$$
x=\mathbf{0 . 5 0}
$$
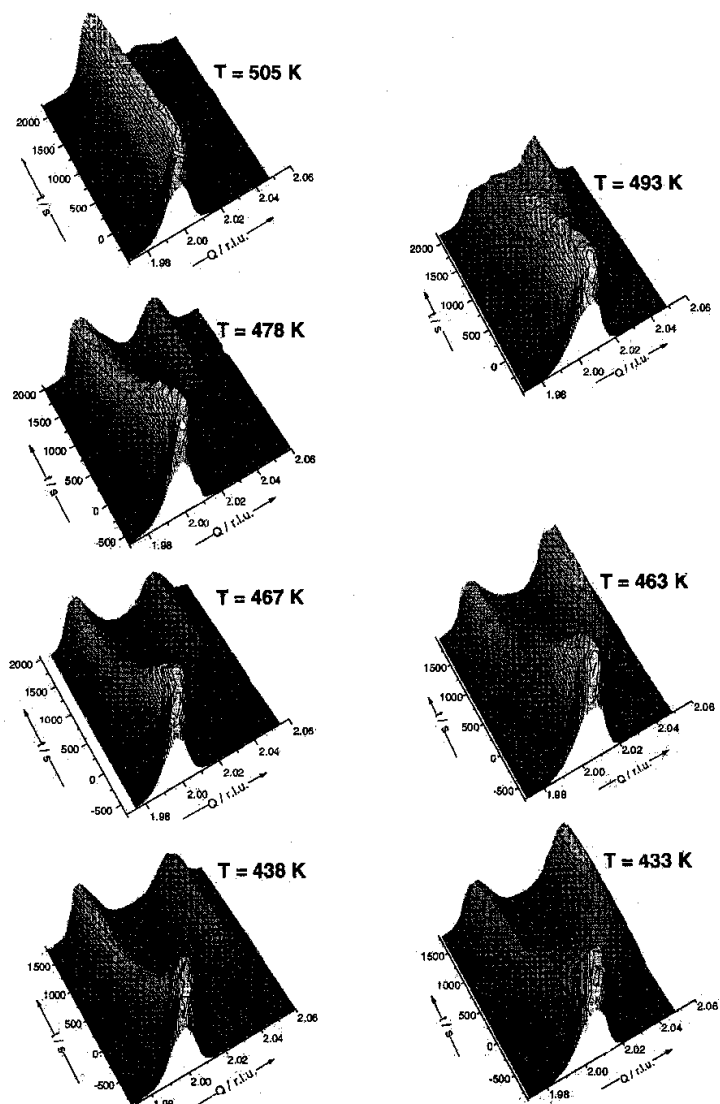

Figure 30: Evolution temporelle du pic de Bragg (200) pour deux compositions $A g_{x} N a_{1-x} B r \dot{a}$ différentes températures [Eckold 2001]. 


\section{LA DIFFRACTION RESOLUE EN TEMPS ET IN SITU SUR MONOCRISTAL}

Ce domaine de la diffusion neutronique est assez peu développé à ce jour car il fut longtemps limité par les détecteurs utilisés (film photographique en géométrie de Laue, faible domaine angulaire couvert par les compteurs de 4 cercles). L'équipement de plus en plus courant de détecteurs bidimensionnels sur les instruments quatre cercles devrait favoriser l'essor des études neutroniques en temps réel effectuées sur monocristal. Ce type d'analyse a déjà été réalisé aux rayons X sur des équipements 4 cercles de laboratoires [Ohashi 1998].

Des travaux pionniers ont été récemment réalisés par une équipe japonaise [Ohhara 1998, Ohashi 1998], qui a pu mener une observation in situ, en temps réel par diffraction neutronique sur monocristal de l'échange hydrogène-deutérium dans un complexe de phényléthylamine. Dans cette expérience originale, un cristal de $3.0 * 3.0 * 0.6 \mathrm{~mm}$ a été illuminé par une lampe fluorescente pendant $36 \mathrm{~h}$. afin de favoriser l'inversion de configuration d'un groupement (R)-1-cyanoéthyle. L'exposition à la lumière permet de casser la liaison $\mathrm{C}-\mathrm{H}$ et favorise l'échange isotopique. Les neutrons apportent ici une information unique sur les mécanismes mis en jeu et ont permis de mettre en évidence la sélectivité de cet échange isotopique qui ne se réalise que sur un groupement spécifique d'une molécule. Ce n'est pas seulement la faisabilité de ces expériences qui a été démontrée mais plusieurs études neutroniques de ce type ont déjà été réalisées par cette équipe [Ohashi 1998]. Ces expériences seront facilitées par l'utilisation de multidétecteurs, qui permettent en outre d'accéder à des échelles de temps plus courtes encore.

Signalons aussi que la décomposition d'un monocristal AgBr- $\mathrm{NaBr}$ comportant $32 \%$ de $\mathrm{AgBr}$ a été réalisée in situ par diffusion neutronique [Winggasse et al. 1997]. Le suivi en temps réel de la réflexion (200) sur un spectromètre 3 axes a permis d'identifier la démixion du composé en deux phases distinctes. Pour cette étude, l'équipe du Pr. Eckold a tiré le meilleur parti de la réversibilité de la réaction (voir figure 30 ).

D'un point de vue expérimental, les avancées significatives ont été réalisées à l'I.L.L. en termes de détection et la conception de diffractomètre de Laue comportant un multidétecteur permet désormais d'envisager des études de diffusion de neutrons sur des monocristaux en des temps de mesures réduits, mesures impensables auparavant. Le temps d'acquisition de ces dispositifs est maintenant nettement réduit, il peut par exemple être de l'ordre de la minute seulement. A titre d'exemple, deux diagrammes de diffraction polychromatique (Laue) sont présentés sur la figure 31. Ainsi les instruments LADI (en fonctionnement) et VIVALDI (en développement) de l'I.L.L. devraient permettre, puisqu'ils sont complémentaires dans la gamme d'énergie de neutrons sélectionnée de couvrir un large domaine de recherche tant en physique, en chimie qu'en biologie. La relative facilité de lecture de leurs détecteurs à base de plaque image permet d'accéder à l'étude de nombreux problèmes. Les temps d'acquisition des diagrammes de la figure 31 sont tout à fait raisonnables, typiquement 5 à 20 minutes. Cependant, la lecture de l'information sur la plaque image occasionne encore un temps mort dans l'acquisition: typiquement deux minutes. Il est donc désormais possible d'effectuer des études de diffraction neutronique de Laue en temps réel, avec une échelle de temps de l'ordre de la minute ce qui est tout à fait raisonnable. Ceci est rendu possible grâce à l'utilisation d'un détecteur cylindrique de $40 \mathrm{~cm}$ de long et $100 \mathrm{~cm}$ de diamètre. L'avantage est ici de disposer en un diagramme d'une large partie du réseau réciproque et donc d'envisager des analyses structurales fines dans un temps réduit. L'instrument VIVALDI en développement à l'I.L.L. sera installé cette année sur un guide de neutrons thermiques et sera donc particulièrement bien adapté aux études de physique et de chimie du solide. En outre, le choix de la configuration verticale du détecteur permet un accès facile à des environnements complexes : cryostat, cellule de pression et autre 

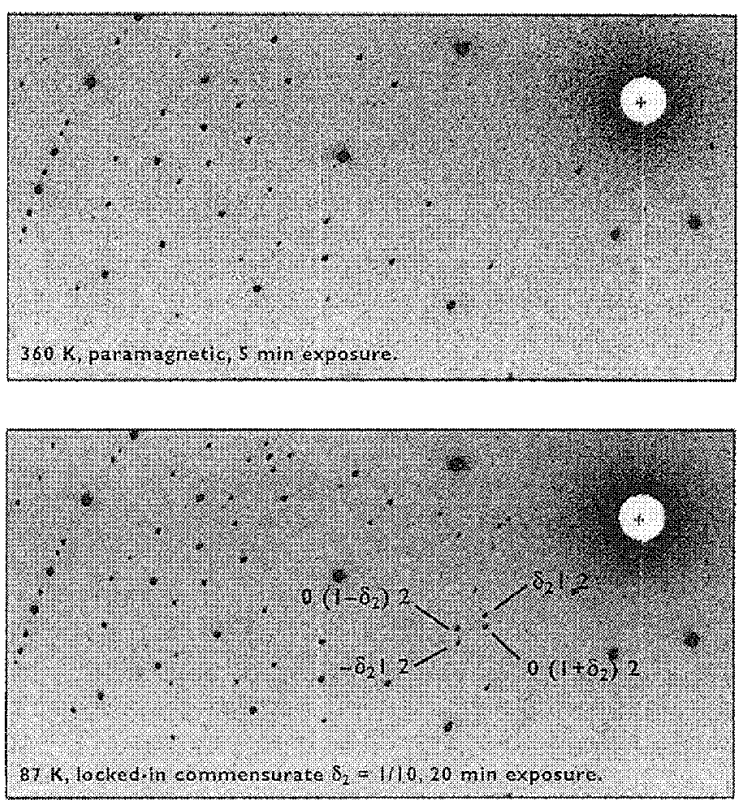

Figure 31 : Diagramme de diffraction de Laue obtenu par C. Wilkinson [Wilkinson 1997] sur le composé $\mathrm{FeGe}_{2}$ dopé avec $4 \%$ de gallium. Le diagramme du haut correspondant à un enregistrement de 5 minutes à $360 \mathrm{~K}$ et celui du bas à un enregistrement de 20 minutes à $87 \mathrm{~K}$. Les différences sont dues à la mise en place d'un ordre magnétique incommensurable caractérisé par des pics de Bragg supplémentaires, Instrument LADI de l'ILL.

\section{LIMITATIONS DE LA DIFFRACTION RESOLUE EN TEMPS}

VIII.a. Echelle de temps caractéristique

Les neutrons sont relativement lents ; leur vitesse s'exprime selon la relation suivante :

$$
\mathrm{V}(\mathrm{mm} / \mu \mathrm{s})=3,955 / \lambda(\AA)
$$

En conséquence, il faut plus de $6 \mu$ s pour un faisceau de neutrons monochromatique $\lambda=2,52 \AA$ pour traverser un échantillon d'un centimètre de diamètre. Cela conduit donc à estimer théoriquement une limite basse de la résolution temporelle d'un spectromètre de neutrons sur source continue à environ $10 \mu \mathrm{s}$. D'un point de vue expérimental, d'autres paramètres interviennent :

- la dispersion en longueur d'onde du faisceau qui conduit à une incertitude sur la durée de vol entre l'échantillon et le détecteur,

- la durée de traversée du détecteur,

- les défauts de synchronisation de l'électronique.

Pour l'instrument D20 à l'I.L.L. la limite expérimentale est estimée par P. Convert à environ $40 \mu \mathrm{s}$ pour une longueur d'onde de $2,5 \AA$, ce qui a été confirmé expérimentalement. 
A titre d'exemple, le diagramme de diffraction de neutrons obtenu en 0,1 seconde sur un échantillon de fer de $8 \mathrm{~mm}(1,28 \AA)$ sur 1'instrument D20 est présenté sur la figure 32 . La figure 33 montre, quant à elle, l'évolution temporelle du diagramme de diffraction de neutrons d'un échantillon de grafoil mis en rotation à 3000 tours par minute sur l'instrument D20. La détection étant synchronisée avec la rotation de l'échantillon, 80 diagrammes de $250 \mu$ s chacun ont été enregistrés par tour c'est-à-dire 80 diagrammes toutes les $20 \mathrm{~ms}$.

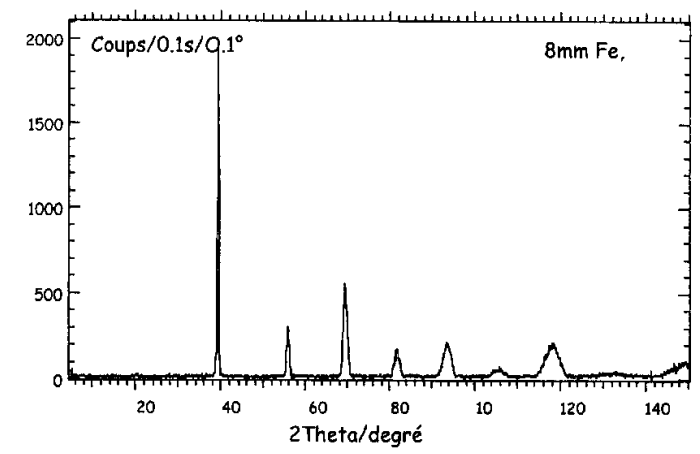

Figure 32 : Diffractogramme neutronique d'un échantillon de poudre de fer mesuré en un dixième de seconde sur l'instrument D20 de l'Institut Laue Langevin.

La relative lenteur du neutron par rapport au rayonnement $X$ ne permet donc pas d'envisager des mesures stroboscopiques ultra-rapides. Ainsi, pour des temps d'acquisition nettement inférieurs à la dizaine de microsecondes, les rayons $\mathrm{X}$ issus du rayonnement synchrotron sont indispensables et peuvent présenter un avantage certain dès des échelles de temps inférieures au dixième de seconde pour l'étude de processus irréversibles.
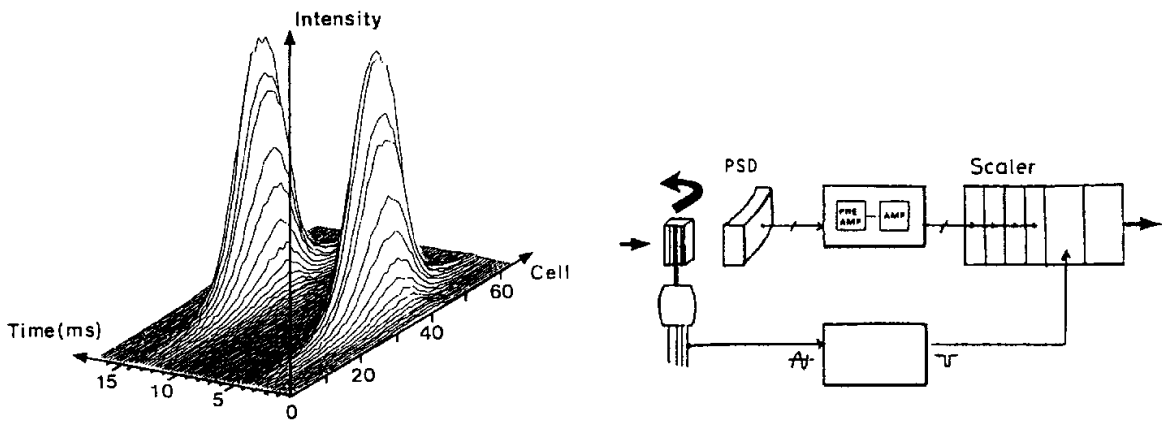

Figure 33 : Diffraction résolue en temps d'un échantillon de grafoil en rotation à 3000t/mn, mesure effectuée sur l'instrument D20 de l'Institut Laue Langevin. L'expérience est schématisée sur la partie droite de la figure [Convert 1997].

Pour conclure, bien que à terme la lenteur du neutron soit un facteur limitant la résolution en temps, cette limite que l'on peut estimer à environ 10 à $50 \mu$ s pour l' instrument D20 à l'I.L.L. n'est pas actuellement le facteur limitant. C'est la nécessité d'un minimum de statistique de comptage qui conduit souvent à des temps d'acquisitions limites de l'ordre de la seconde. Il y a 
encore place pour des améliorations sensibles soit en terme de flux soit en terme d'angle solide de détection. Concernant les sources à spallation, un gain significatif en terme de flux est attendu suite au développement de nouvelles sources de neutron telles que l'European Spallation Source. Les multidétecteurs actuellement utilisés sur D1B ou D20 sont imposants mais ils ne couvrent finalement qu'une faible partie de l'angle solide de diffraction si on les compare aux détecteurs utilisés sur d'autres instruments. Il y a là encore un potentiel de développement important.

Rappelons que dans nombre de cas comme pour la thermodiffractométrie, il est possible d'ajuster l'échelle de temps à la sonde utilisée (ex : via le choix de la rampe thermique). L'échelle de temps ne doit pas être prise comme un critère de comparaison absolu, en effet bien d'autres critères sont à prendre en compte parmi lesquels :

- le contraste atomique ou isotopique,

- le flux,

- la résolution,

- le pouvoir diffusant de l'échantillon, la quantité d'échantillon,

- la sensibilité de la sonde neutrons ou rayons $\mathrm{X}$ au problème donné est à comparer,

- le domaine de température requis peut nécessiter l'utilisation d'un rayonnement plutôt qu'un autre,

- l'environnement échantillon nécessaire, ex: dimension, complexité des cellules électrochimiques,

- et bien entendu le point déterminant est la question à laquelle on souhaite répondre, veut-on sonder le volume? Veut-on sonder la surface de l'échantillon ?...

Tous ces critères peuvent, selon les expériences, être aussi sinon plus importants que celui de l'échelle de temps. En outre, nous avons vu que si l'on étudie un phénomène réversible, une technique relativement lente comme la spectrométrie 3 axes neutrons peut cependant être utilisée pour caractériser en temps réel des phénomènes très rapides allant jusqu'à $10^{-3} \mathrm{~s}$. Ainsi, l'utilisation des techniques neutroniques peut largement être étendue à des échelles de temps courtes lorsque le phénomène est réversible et peut donc être répété.

Nous avons déjà évoqué un certain nombre de limitations instrumentales aux études résolues en temps, d'autres sont inhérentes au fait que inévitablement, une étude en temps réel est hors équilibre. Ainsi la taille des échantillons utilisés peut être la source d'inhomogénéités de température lors d'une expérience de thermodiffractométrie. Il faut alors réduire la vitesse de rampe thermique afin de s'assurer du quasi équilibre de l'échantillon; cela se fait naturellement au détriment de la vitesse d'acquisition recherchée. D'autres sources d'inhomogénéité peuvent apparaitre : des transformations structurales peuvent induire des défauts ou des contraintes dans le matériau. Cela peut affecter les profils de pics de diffraction, se traduire par des élargissements de pics ce qui est équivalent à une perte en résolution. Lors de l'étude de réactions chimiques, il peut $\mathrm{y}$ avoir nécessité de transport de matière et donc des gradients de composition. Cela conduit donc à une inhomogénéité qui peut être d'autant plus élevée que la cinétique de réaction choisie est grande. L'étude par diffusion de neutrons permet alors d'avoir une information moyenne sur l'échantillon puisque les neutrons sondent le volume de celui-ci. Cette information ne sera plus caractéristique d'un état pseudo-équilibre de tout l'échantillon mais traduira vraiment une moyenne des différents états de l'échantillon. Il est alors possible de bénéficier de la complémentarité entre rayons $\mathrm{X}$ et neutrons : dans le cas de la diffraction des rayons $\mathrm{X}$, c'est généralement la surface de l'échantillon qui sera sondée même si l'utilisation de rayons $\mathrm{X}$ de haute énergie ( synchrotron) permet de disposer de faisceau plus pénétrant. En revanche, le caractère pénétrant des neutrons sera particulièrement apprécié pour les études en volume de l'échantillon. Enfin la facilité avec laquelle il est possible de focaliser un faisceau de rayons $X$ peut permettre de choisir de sonder différentes parties de l'échantillon et donc de sonder sélectivement différentes zones pour vérifier l'homogénéité de celui ci. 
D'autres limites telles que la vitesse de diffusion des atomes ou des molécules au cours d'une réaction chimique peuvent aussi jouer un rôle important.
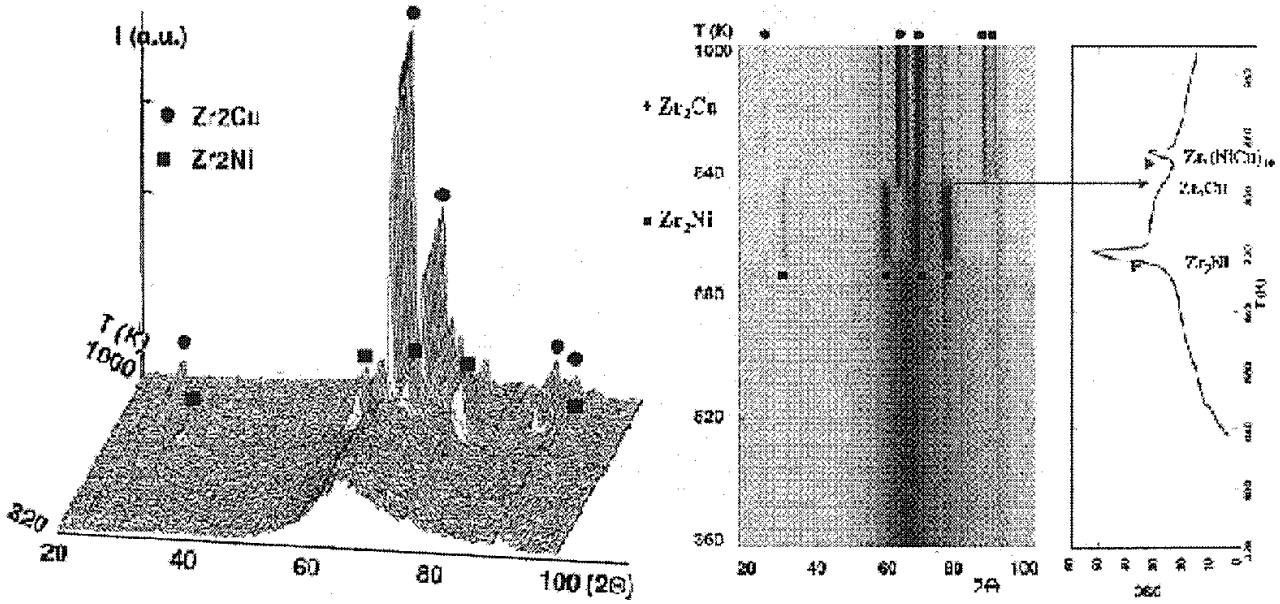

Figure 34 : Thermodiffractogramme de neutrons enregistré en temps réel lors de la cristallisation d'un alliage amorphe. Instrument D1B de l'I.L.L. Les carrés et les ronds repèrent les pics de Bragg correspondant aux phases $\mathrm{Zr}_{2} \mathrm{Ni}$ et $\mathrm{Zr}_{2} \mathrm{Cu}$ respectivement. Le diffractogramme est représenté sur la gauche de la figure, le tracé de contour correspondant est au centre de la figure et comparé au signal de D.S.C. enregistré dans les mêmes conditions. La phase $\mathrm{Zr}_{7}(\mathrm{NiCu})_{10}$ apparaît ensuite au dessus de 580C.

\section{VIII.b. Développements en cours}

Comme nous venons de le voir, d'importants progrès ont été réalisés ces dernières années en diffusion de neutrons en temps réel : citons la mise en service de nouveaux instruments tels que D20 à l'ILL ou l'apparition de GEM (General Materials Diffractometer) à ISIS qui a permis de démontrer la faisabilité de ce type d'étude sur une source à spallation. Les développements peuvent encore être nombreux, l'utilisation du temps de vol sur les nouvelles sources à spallation peut permettre de tirer parti de l'utilisation d'un faisceau polychromatique (sans la perte de flux occasionnée par un monochromateur). Tout comme le faisceau synchrotron, une source à spallation produit des neutrons de manière discontinue avec une fréquence typique de l'ordre de $50 \mathrm{~Hz}$. Dès lors, il est envisageable de tirer parti de cette structure temporelle du faisceau pour réaliser des études en temps réel de type stroboscopique.

Parmi les améliorations souhaitables, il est bien sûr espéré de pouvoir étendre la gamme de temps accessible (cf figure 35), mais aussi de développer de nouvelles applications pour la diffusion en temps réel. Pour cela, l'interaction entre la communauté des utilisateurs et les responsables d'instrument sera encore déterminante. L'étude de systèmes toujours plus complexes s'enrichira de mesures simultanées ( pression, température etc...) qui associées à la diffusion en temps réel permettent de compléter les informations purement structurales. Cela est réalisé avec succès au travers de la mesure du potentiel et du courant dans les études électrochimiques. D'autres informations peuvent elles aussi être combinées à la diffraction neutronique en temps réel. Ainsi le développement par le CRG CNRS-D1B d'une balance thermogravimétrique permettant de travailler in situ et sous atmosphère gazeuse contrôlée permet de mieux suivre les réactions solide gaz. 
Dans le cas de la cristallisation de verres métalliques massifs à base de zirconium, la confrontation des mesures de DSC avec les résultats de diffraction neutronique ont permis de mieux comprendre les étapes de la recristallisation voir figure 34 . On observe clairement à $425^{\circ} \mathrm{C}$ l'apparition de pics de diffraction qui correspondent aux pics de cristallisation observés. Cette démarche utilisant en simultané des techniques complémentaires de la diffraction neutronique est à développer.

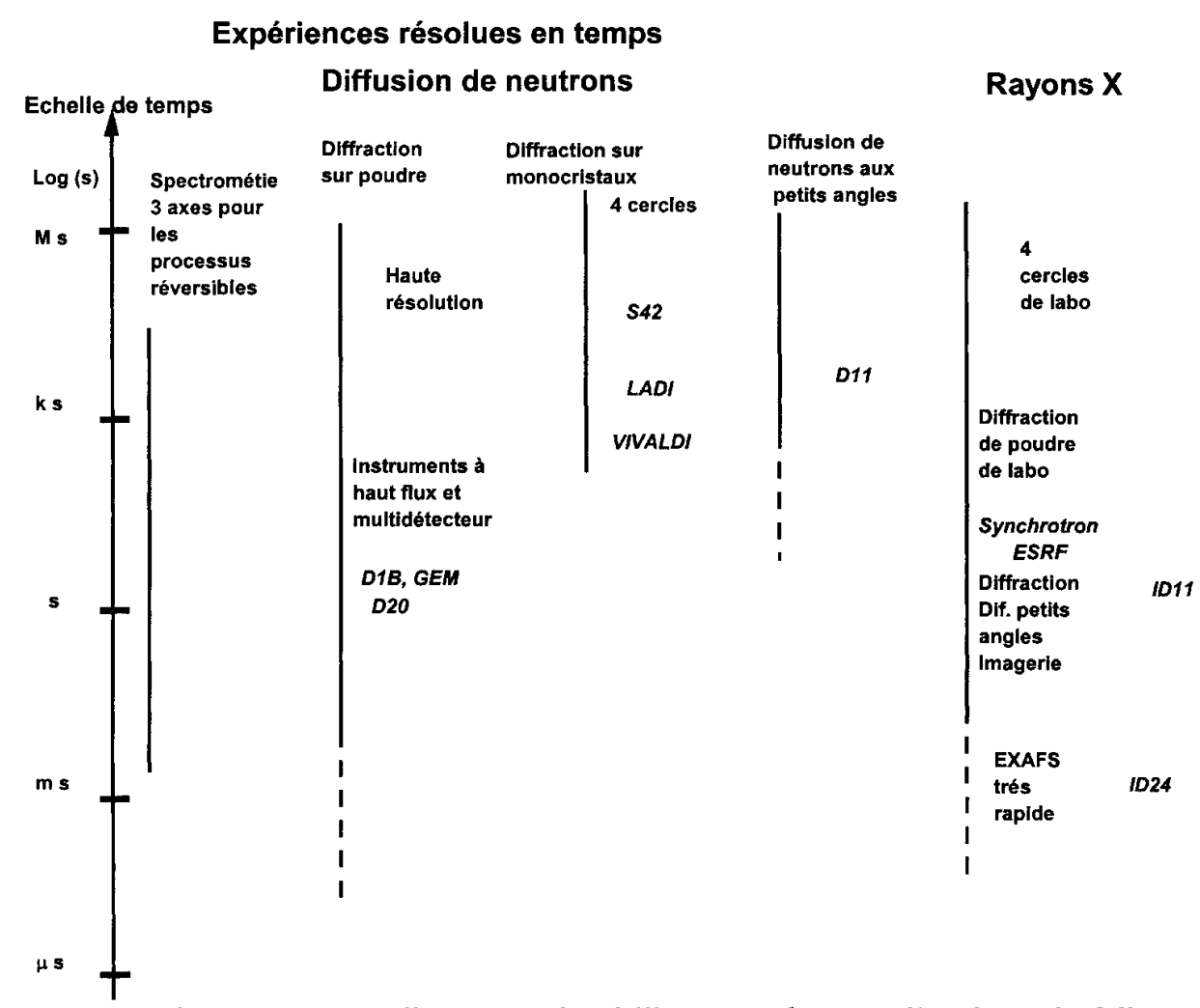

Figure 35 : Résolution temporelle typique des différentes techniques d'analyses de diffusion de neutrons en temps réel. Les domaines classiques d'études sont indiqués en trait plein et ceux accessibles le sont en pointillé. A titre de comparaison, une échelle est donnée sur la figure de droite pour les rayons $X$. Tous les domaines représentés ici font référence aux analyses irréversibles à l'exception de la spectroscopie trois axes qui fait référence aux travaux de Eckold [Eckold et al. 1990] sur des processus réversibles.

\section{CONCLUSIONS}

L'utilité de la diffusion de neutron en temps réel a été présentée sur la base d'exemples récents. Les possibilités actuelles et le potentiel de cette technique d'investigation dynamique ont été discutés. Ce type d'analyse permet de manière non destructive d'obtenir une information directe sur les processus mis en jeu lors des réactions chimiques ou des transformations de la matière. Ces analyses en temps réel peuvent, grâce à la faible absorption des neutrons, être réalisées in situ et apportent une meilleure compréhension de la complexité des phénomènes mis 
en jeu. Ces études en temps réel sont donc très largement utilisées par diverses communautés scientifiques : sciences des matériaux, physiciens, chimistes...

Pour les expérience résolues en temps, plus qu'ailleurs il est nécessaire de faire un compromis entre temps d'acquisition et résolution. C'est particulièrement vrai pour les neutrons puisque les flux disponibles sont relativement faibles en regard de ceux disponibles sur d'autres grands instruments. L'optimisation des conditions de collecte dépend non seulement de l'intensité du faisceau incident mais aussi du pouvoir diffractant de l'échantillon à la longueur d'onde utilisée et enfin de l'efficacité du détecteur qui est elle aussi fonction de l'énergie du rayonnement. La comparaison des performances de divers instruments ne peut se limiter à la comparaison des flux incidents, mais il faut aussi tenir compte des longueurs d'ondes disponibles et des résolutions accessibles...

Ce type d'expérience résolue en temps est aussi réalisable par diffraction de rayons $\mathrm{X}$ en particulier à partir de rayonnement synchrotron. Le choix d'un rayonnement doit être fait en fonction du problème à résoudre et de l'échelle de temps du phénomène étudié. Les neutrons offrent l'avantage de sonder la matière en volume et sont incomparables pour l'étude du magnétisme ou les études structurales de matériaux comportant à la fois des éléments légers et des éléments lourds. Bien que la plupart des travaux actuels soient caractérisés par un temps d'acquisition de l'ordre de la minute, il est déjà possible d'effectuer des études de diffusion de neutrons en temps réel dans la gamme de la seconde et nettement en dessous. Rappelons qu'une estimation des échelles de temps caractéristiques, accessibles par diverses techniques neutroniques, est indiquée dans la figure 35 .

Le domaine de prédilection des études en temps réel est la diffraction neutronique sur échantillon polycristallin, cependant la faisabilité de ce type d'analyse a été démontrée pour nombre d'autres techniques neutroniques : diffusion au petits angles, diffraction sur monocristaux, spectrométrie trois axes... et le fort potentiel de ces méthodes d'analyses en temps réel n'attend que les bonnes idées de recherche.

\section{REFERENCES}

Anne M., Boudjada N., Rodriguez J., Figlarz M., Inst. Phys. Conf. Ser. 107 chap. 2, Institute of Physics Publishing (1990) 117-125.

Ballon J., Comparat V., Pouxe J. Nucl. Instr. Meth. 217 (1983) 213.

Barret P., Gerard N., Watelle-Marion G. Bull. Soc. Chim. Fr. 8 (1968) 3172.

Bellet D., Bastie P., Royer A., Lajzerovicz J., Legrand J.F., Bonnet R. J. Phys. I, France 2, 1097 (1992).

Bellet D. Thèse de 1'Université Joseph Fourier, Grenoble (1990)

Bellet D., Royer A. , Bastie P. Lajzerowicz J., Legrand J.F., seven International Symposium on superalloys, seven Spings Champion USA (1992) Superalloys 92, (1992) 547-556

Bellet D., Contributions des techniques neutroniques à l'étude des superalliages monocrystallins, Ecole de printemps diffusion des neutrons et sciences des matériaux 21-27 Avril 1997, Pinsot.

Boudjada N., Rodriguez-Carvajal J., Anne M., Figlarz M. J. of Sol. State Chem. 105, 211-222 (1993). 
Brianso M.C. et Isnard O., Structures magnétiques support pédagogique consultable sur le site http://www-llb.cea.fr

Chabre Y., Pannetier J., Progress Solid State Chem. 23 (1995) 1-130

Chabre Y., Bardé F., Palacin R., Isnard O., Tarascon J.M. rapport d'expérience CRG-D1B 2001.

Chabre Y., «Electrochimie des composés d'Insertion » Chapitre XII, à paraître(2002)

Chabre Y. NATO ASI « Chemical Physic of intercalation II», Bonas, France, Juillet 1992 ; NATO Series , B305, 181-92 (1993).

Chappel E., Nunez-Regueiro M.D., Chouteau G., Isnard O., Darie C., Eur. Phys. J. B 17, (2000) 615-622.

Chuang-Dong, de Boissieu M., Dubois J.M., Pannetier J., Janot C.,J. Materials Science Lett. 8, (1989) 827-830.

Chenevier B., Thèse, Université Joseph Fourier, Grenoble I

Convert P., Thèse, Université de Grenoble (1975)

Convert P., Fruchart D., Roudaut, E. et Wolfers P., dans Position Sensitive Detection of Thermal (ed. Convert P. et Forsyth J.B.) 302, Academic Press (1983).

Convert P., Notes de cours à l'école thématique «Cristallographie et Neutrons » Batz sur mer 1997.

Convert P., Hansen T., Oed A., Toregrossa J. Physica B 241-243 (1998) 195-197.

Cotton J.P., Nallet F. «Diffusion des neutrons aux petits angles (DPNA) » (1999) EDP Sciences.

Debye P. Ann. Physik, 46, 809, 1915.

Eckold G., Nucl. Instr. Meth. A238 (1990) 221-230.

Eckold G., Trzecciok D., Physica B 180-181 (1992) 315-317.

Eckold G., J. Phys. Condens. Matter 13 (2001) 217-240.

Fruchart D., J.L. Soubeyroux, S. Miraglia, S. Obbade, G. Lorthioir, F. Basile, C. Colin, F. Faudot, P. Ochin, A. Dezellus, Zeitschrift für Physikalische Chemis 179 (1993) 225-236.

Gibhardt H., Eckold G., Güthoff F., Physica B 276-278 (2000) 240-241.

Goldmann M., Pannetier J., Beguin F., Gonzalez F., Synthetic Metals 23, (1998) 133-138.

Goldman M., Pannetier J., Beguin F., Phase transitions 14 (1989) 153-159.

Goutenoire F., Isnard O., Suard E., Bohnke O., Laligant Y., Retoux R. et Lacorre Ph., J. Mater. Chem., 11(2001) 119-124.

Gerold V. et Kostorz G. J. Appl. Cryst 11 (1978) 376-404. 
Guinier A., Théorie et technique de la radiocristallographie, chap 10 et 11 Dunod éd., 1964.

Ishikawa Y., Arai M. , Furusaka M. et Mera M. KENS report IV KEK internal report 83-4.

Isnard O., Soubeyroux J.L., Miraglia S., Fruchart D., Garcia L.M., Bartolome J., Physica 180181B (1992) 624-626.

Isnard O., Soubeyroux J.L., Miraglia S., Fruchart D., Garcia L.M., Bartolome J., Physica 180181B (1992) 629-631.

Janot C., Dubois J.M., «Les quasicristaux. Matière à paradoxes » collection monographie de Matérialogie (1998) EDP Sciences

Kilcoyne S.H., Manuel P., Ritter C. Padaelli P. ILL millenium symposium 6-7 Avril 2001

Lacorre Ph., Goutenoire F., Isnard O., Suard E., I.L.L. Annual Report (2000), 38-39.

Latroche M., Percheron-Guégan A. and Chabre Y. J. Alloys Comp. 293-295 (1999) 637-642.

Latroche M. , Chabre Y., Percheron Guegan A., Decamps B . Noreus N., soumis à J. Alloys and Comp. (2001).

Latroche Y., Percheron-Guegan A., Chabre Y. O. Isnard, à paraître J. Alloys and Comp. (2001).

Menarde M., Rodriguez J., Vallet M., Pernet M., Obradors X. et Pannetier J. 156-157 Physica B (1989) 36.

Notten P.H.L. Daams J.L.C., De Veiram A.E.M. Staals A.A., J. Alloys and Comp. 209 (1994) 8591.

Ohhara T., Uekusa H., Ohasahi Y., Tanaka I., Kumasawa S., Niimura N. Chem. Lett. (1998) 365366

Ohashi Y., Acta. Cryst. A54 (1998) 842-849.

Oleinek Ph. Thèse Université de Dresde Allemagne 2000

Oleinek Ph., Isnard O., Convert P., Müller K.H., Loewenhaupt M., Schultz L., J. Alloys and Comp. 298 (2000) 220-225.

Pannetier J., Chem. Scripta 26A (1986) 131

Pelletier J.M., J. Perez, Soubeyroux J.L., J. Non-Cryst. Solids, 274 (2000) 301-306.

Pillière H., Thèse de l'Université d'Orléans, (1991)

Plévert J., Audeffric J.P., Louër M., Louër D. J. Mater. Sci. 24 (1989) 1913.

Rippert M. , Pannetier J. Chabre Y. Poinsignon C. Mat. Res. Soc. Symp. Proc. 210 (1991) 359365.

Rippert M. Thèse INPG (1990) 
Rigny, P. Dianoux A.J. l'actualité chimique Février (1979) 7-18.

Rodriguez J., Bassas J., Obradors X., Vallet M. Calbet J., M. Anne, Pannetier J. Physica C 153155 (1988) 1671-1672.

Schweitzer J., Chapitre 5 et 6, dans Neutron and Synchrotron Radiation for Condensed Matter Studies, HERCULES courses, volume II, ed. J. Baruchel, J.L. Hodeau, M.S. Lehmann, J.R. Regnard, C. Schlenker, EDP Sciences (1994)

Seguin L., Thèse Université de Picardie, (1994)

Soubeyroux J.L., Pelletier J.M., Perrier de la Bâthie R., Physica B 276-278 (905-906)

Soubeyroux J.L., Claret N, rapport d'expérience sur le CRG-D1B (2001)

Vannier R.N., Abraham F., Nowogrocki G., Mairesse G., J. Solid State Chem. 142 (1999) 294304.

Veron M., Bastie P. Acta Materiala 45 (1997) 3277-3288.

Wilkinson C. et al. Annual Report 97 Institut Laue Langevin.

Wilkinson C., Mc Intyre C.J., Cowan J.A., Myles D.A.A., Lehmann M.S., à paraître J. Appl. Cryst.

Williams W.G., R.M. Iberson, P. Day, J.E. Enderby, Physica B 241-243 (1998) 234-236

Winggasse J., Eckold G., Güthoff F., Physica B 234-236 (1997) 153-154.

Wong Joe, E.M. Larson, Holt J.B., Waide P.A., Rupp B, FRAHM R. Science 249 (1990) 14061409.

\section{Autres références utiles :}

Time-resolved diffraction, Oxford Series in Synchrotron Radiation 2, J.R. Helliwell et P.M. Rentzepis, Oxford Science Publications

J. Pannetier, 1988, "Real time neutron powder diffraction", dans Chemical Crystallography with pulsed neutrons and Synchrotron X-rays, edité par M.A. Carrondo et G.A. Geffrey, NATO ASI series C vol 221 (1988) p313-355, D. Reidel Publishing and Co

« Time resolved studies » dans Handbook of synchrotron radiation, vol. 3, édité par G. Brown et D.E. Moncton, Elsevier Science Publishers B.V.

Pannetier J., "Time resolved scattering experiments", Chap. XIX, dans neutron and synchrotron radiation for conden,sed matter studies vol. I, édité par J. baruchel, J.L. Hodeau, M. Lehman, J.R. Regnard, C. Schlenker, Les éditions de Physiques 1993.

Moron M.C., J. Mater. Chem. 10 (2000)2617-2626.

Pannetier J. Chemica Scripta 26A (1986) 131-139.

Rodriguez Carvajal J. Physica B 192 (1993) 55-69. 


\section{Sites à visiter sur la toile :}

http:/www. ill.fr

http:/www.sfn.asso.fr

http:/www-llb.cea.fi/cours/cours.html

http://www-llb.cea.fr/fullweb

http//www-cristallo.polycnrs-gre.fr

\section{Remerciements}

Je remercie les gens qui m'ont appris la diffraction des neutrons au premier rang desquels Jean Pannetier et Pierre Convert qui ont tant œuvré pour le développement de ces techniques de diffusion en temps réel.

Les discussions avec les collègues : G. Eckold, P. Bastie, M. Anne, P. Radaelli, T. Hansen m'ont été aussi agréables qu'utiles, je les en remercie.

Comme tout responsable d'instrument, mon activité a été stimulée par le contact et les échanges avec divers utilisateurs avertis et ouverts d'esprit, je les remercie donc tous et plus particulièrement, Y. Chabre, M. Latroche. 